\title{
Effects of prehabilitation and rehabilitation including a home-based component on physical fitness, adherence, treatment tolerance, and recovery in patients with non-small cell lung cancer
}

Citation for published version (APA):

Driessen, E. J., Peeters, M. E., Bongers, B. C., Maas, H. A., Bootsma, G. P., van Meeteren, N. L., \& Janssen-Heijnen, M. L. (2017). Effects of prehabilitation and rehabilitation including a home-based component on physical fitness, adherence, treatment tolerance, and recovery in patients with non-small cell lung cancer: A systematic review. Critical Reviews in Oncology/Hematology, 114, 63-76. https://doi.org/10.1016/j.critrevonc.2017.03.031

Document status and date:

Published: 01/06/2017

DOI:

10.1016/j.critrevonc.2017.03.031

Document Version:

Publisher's PDF, also known as Version of record

\section{Document license:}

Taverne

\section{Please check the document version of this publication:}

- A submitted manuscript is the version of the article upon submission and before peer-review. There can be important differences between the submitted version and the official published version of record. People interested in the research are advised to contact the author for the final version of the publication, or visit the DOI to the publisher's website.

- The final author version and the galley proof are versions of the publication after peer review.

- The final published version features the final layout of the paper including the volume, issue and page numbers.

Link to publication

\footnotetext{
General rights rights.

- You may freely distribute the URL identifying the publication in the public portal. please follow below link for the End User Agreement:

www.umlib.nl/taverne-license

Take down policy

If you believe that this document breaches copyright please contact us at:

repository@maastrichtuniversity.nl

providing details and we will investigate your claim.
}

Copyright and moral rights for the publications made accessible in the public portal are retained by the authors and/or other copyright owners and it is a condition of accessing publications that users recognise and abide by the legal requirements associated with these

- Users may download and print one copy of any publication from the public portal for the purpose of private study or research.

- You may not further distribute the material or use it for any profit-making activity or commercial gain

If the publication is distributed under the terms of Article $25 \mathrm{fa}$ of the Dutch Copyright Act, indicated by the "Taverne" license above,

Download date: 26 Apr. 2023 


\title{
Effects of prehabilitation and rehabilitation including a home-based component on physical fitness, adherence, treatment tolerance, and recovery in patients with non-small cell lung cancer: A systematic review
}

\author{
Elisabeth J. Driessen ${ }^{a, *}$, Marieke E. Peeters ${ }^{b}$, Bart C. Bongers ${ }^{c}$, Huub A. Maas ${ }^{d}$, \\ Gerbern P. Bootsma ${ }^{\mathrm{e}}$, Nico L. van Meeteren ${ }^{\mathrm{c}, \mathrm{f}}$, Maryska L. Janssen-Heijnen ${ }^{\mathrm{a}, \mathrm{g}}$ \\ a Department of Clinical Epidemiology, VieCuri Medical Centre, Venlo, The Netherlands \\ ${ }^{\mathrm{b}}$ Department of Physical Therapy, Knooppunt Centre for Health, Baarlo and Venray, The Netherlands \\ c Department of Epidemiology and Research School Caphri, Maastricht University, Maastricht, The Netherlands \\ d Department of Geriatric Medicine, Elisabeth-Tweesteden Hospital, Tilburg, The Netherlands \\ e Department of Pulmonology, Zuyderland Medical Centre, Heerlen, The Netherlands \\ ${ }^{\mathrm{f}}$ Top Sector Life Sciences \& Health/Health Holland, The Hague, The Netherlands \\ ${ }^{g}$ Department of Epidemiology, Maastricht University Medical Centre, GROW School for Oncology and Developmental Biology, Maastricht, The Netherlands
}

\section{Contents}

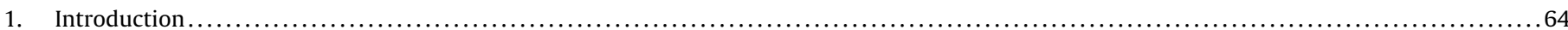

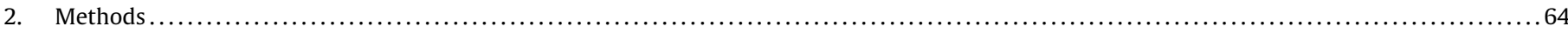

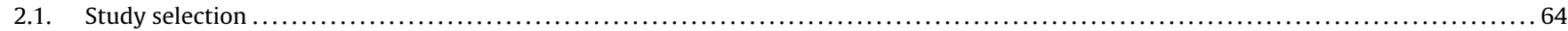

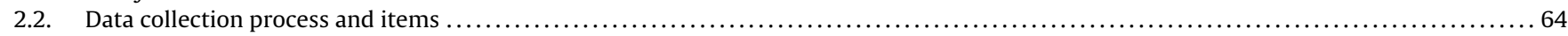

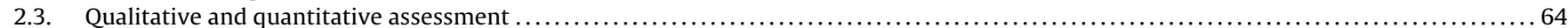

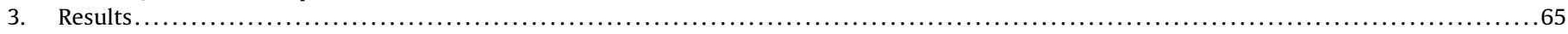

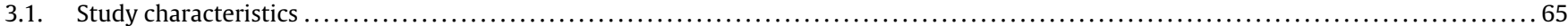

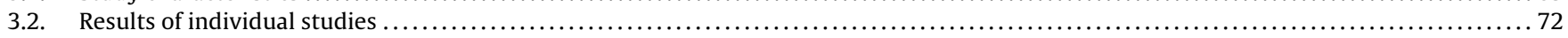

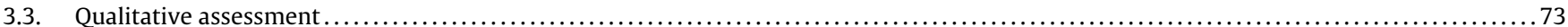

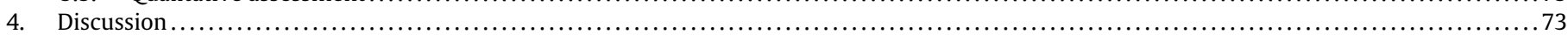

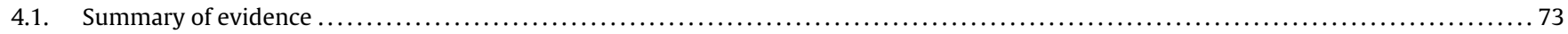

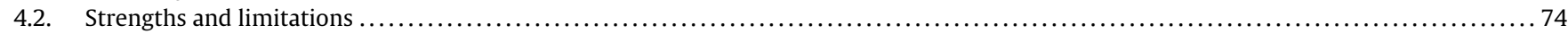

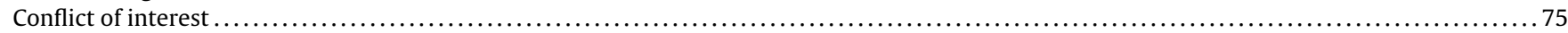

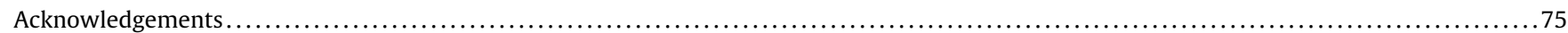

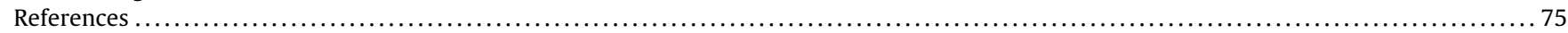

\section{A R T I C L E I N F O}

\section{Article history:}

Received 19 December 2016

Received in revised form 22 March 2017

\section{Keywords:}

Non-small cell lung cancer

Prehabilitation

Rehabilitation

\begin{abstract}
A B S T R A C T
This systematic review aimed to examine physical fitness, adherence, treatment tolerance, and recovery for (p)rehabilitation including a home-based component for patients with non-small cell lung cancer (NSCLC). PRISMA and Cochrane guidelines were followed. Studies describing (home-based) prehabilitation or rehabilitation in patients with NSCLC were included from four databases (January 2000-April 2016, $N=11$ ). Nine of ten rehabilitation studies and one prehabilitation study (437 NSCLC patients, mean age 59-72 years) showed significantly or clinically relevant improved physical fitness. Three (27\%) assessed home-based training and eight (73\%) combined training at home, inhospital (intramural) and/or at the physiotherapy practice/department (extramural). Six (55\%) applied supervision of home-based
\end{abstract}

\footnotetext{
* Corresponding author at: Department of Clinical Epidemiology, VieCuri Medical Centre, P.O. Box 1926, 5900 BX Venlo, The Netherlands.

E-mail addresses: Idriessen@viecuri.nl, research@viecuri.nl (E.J. Driessen), mariekepeeters13@hotmail.com (M.E. Peeters), bart.bongers@maastrichtuniversity.nl (B.C. Bongers), h.maas@etz.nl (H.A. Maas), g.bootsma@zuyderland.nl (G.P. Bootsma), meeteren@health-holland.com (N.L. van Meeteren), mjanssenheijnen@viecui.nl (M.L. Janssen-Heijnen)
} 
Physical therapy

Compliance

Tolerance

Home components, and four (36\%) a personalized training program. Adherence varied strongly (9-125\% for exercises, $50-100 \%$ for patients). Treatment tolerance and recovery were heterogeneously reported. Although promising results of ( $p$ )rehabilitation for improving physical fitness were found (especially in case of supervision and personalization), adequately powered studies for home-based (p)rehabilitation are needed.

(C) 2017 Elsevier B.V. All rights reserved.

\section{Introduction}

Non-small cell lung cancer (NSCLC) concerns 85\% of all lung cancer patients (Netherlands Cancer Registry, 2016a). Five-year survival rates remain low ranging from $55 \%, 41 \%, 13 \%$, to $2 \%$ for stage I, II, III and IV, respectively (Netherlands Cancer Registry, 2016b). Standard curative treatments, including lung resection (Vansteenkiste et al., 2014) and concurrent chemoradiation (Eberhardt et al., 2015), lead to adverse events in $\geq 50 \%$ of patients and frequently require hospitalization (Janssen-Heijnen et al., 2004; Schild et al., 2003). High age (Netherlands Cancer Registry, 2016c), smoking-related comorbidities, frailty, poor performance status, and long-term physical inactivity are often present in patients with NSCLC (Janssen-Heijnen et al., 2004; Hsu et al., 2015; Semrau et al., 2014; Granger et al., 2014). These characteristics may affect mobility, independence, treatment tolerance, recovery, and prognosis (Gridelli et al., 2007; Cardenal et al., 2015; Glotzer et al., 2013; Kale et al., 2015; Hoogeboom et al., 2014). Resistance and endurance training can increase the functional and physiological reserve, thereby creating a safety margin to meet potential enlarged demands of cardiac output and other physical capacities at the time of disease and interventions (Hoogeboom et al., 2014; Carli and Zavorsky, 2005). Prehabilitation (therapeutic training before undergoing treatment) (Carli and Zavorsky, 2005) and rehabilitation (therapeutic training during and after treatment) (Spruit et al., 2013) can optimize physical fitness, treatment tolerance, recovery, and survival (Singh et al., 2013; Ni et al., 2016; Bade et al., 2015), even in older cancer patients (Chou et al., 2012; Jack et al., 2011; Kilari et al., 2016). However, intramural training (inhospital) or extramural training (at the physiotherapy practice or department) may counteract compliance of high-risk patients because of commuting problems, accessibility of services, multimorbidity, and vulnerability (Temel et al., 2009; Oosting et al., 2012). A personalized training program in a home-based setting might overcome these barriers and enhance both motivation and adherence, especially for vulnerable and older patients (Bade et al., 2015). Therefore, the aim of this study is to systematically review the literature regarding feasibility and effectiveness of prehabilitation and rehabilitation including a home-based component in patients with NSCLC by evaluating physical fitness, and to describe adherence and treatment tolerance, and recovery.

\section{Methods}

The Cochrane guidelines for systematic reviews (The Cochrane Collaboration, 2011) and PRISMA guidelines (Preferred Reporting Items for Systematic Reviews and Meta-Analyses) (Moher et al., 2009) were followed. Databases Pubmed, Medline, Embase, and PEDro were searched for eligible articles describing patients with NSCLC undergoing prehabilitation or rehabilitation including a home-based component focusing on physical fitness (Table 1). Search terms were explored on free text words to avoid exclusion of recently published articles. Inclusion was limited to studies in English or Dutch language between January 1, 2000 and April 11,2016 . The primary outcome was physical fitness and secondary outcomes were patient adherence, exercise adherence, treatment tolerance, and recovery. Studies were excluded when insufficient training information was available to distinguish prehabilitation and rehabilitation, and when no physical intervention was applied.

\subsection{Study selection}

All search results were combined and duplicates removed. Assessment of title, abstract and full-texts according to eligibility criteria was performed independently by reviewers MP and ED. Inclusion of eligible studies was discussed until consensus. If no consensus was reached, a third person (BB) determined eligibility. Studies were included when full-text was available. Reference tracking was performed after full-text assessment in order to include additional relevant studies.

\subsection{Data collection process and items}

For each included article, the following information was independently collected, compared, and combined: first author, publication year, study type (prehabilitation/rehabilitation, country, type of study, randomization), demographics (number of patients with NSCLC, stage of disease, age, sex, treatment, comorbidity, performance status), description of the intervention (exercise content, frequency, intensity, measurement times, exercise time, follow-up, time of delivery, controls), and outcomes (physical fitness, patient adherence, exercise adherence, treatment tolerance, recovery). Physical exercises consisted of resistance and endurance training, and training effects were mainly evaluated by the 6-minute walk test (6MWT) distance. Results were described as mean \pm standard deviation, mean (range), or mean difference \pm standard deviation. A minimal clinically important gain of $\geq 42 \mathrm{~m}$ or $9.5 \%$ change was considered clinically relevant for $6 \mathrm{MWT}$ distance (Granger et al., 2015). Patient adherence was described; both patient and exercise adherence (percentage) were considered sufficient above $70 \%$. Treatment tolerance and recovery were displayed by adverse events (numbers, including postoperative complications) and hospitalization time (days, mean \pm standard deviation). Differences between outcomes were considered statistically significant if $P<0.05$.

\subsection{Qualitative and quantitative assessment}

Methodological quality was independently assessed by using the domain-based evaluation for systematic reviews by the Cochrane 'Risk of bias tool' (Cochrane Statistical Methods Group and Cochrane Bias Methods Group, 2011a). Selection-, performance-, detection-, attrition-, and reporting bias were scored present $(+)$ or absent $(-)$. Low, moderate, or high risk of bias was determined by the percentage of present bias, corresponding to high ( $\leq 17 \%)$, moderate (18-33\%), or low ( $\geq 50 \%$ ) methodological quality, respectively. Therapeutic validity for quality of the training content was assessed by the CONTENT scale (Consensus on Therapeutic Exercise Training) (Hoogeboom et al., 2012; Herbert and $\mathrm{B} \varnothing, 2005)$. Nine items regarding patient eligibility, competences and setting, rationale of the study, content of the study, and adherence were scored as performed $(+)$ or not performed 
Table 1

Combinations of text words per database of the literature search.

\begin{tabular}{|c|c|c|c|}
\hline Databases & $\begin{array}{l}\text { Population } \\
\text { Non-small cell lung cancer patients }\end{array}$ & $\begin{array}{l}\text { Intervention } \\
\text { Home-based }(p) \text { rehabilitation }\end{array}$ & $\begin{array}{l}\text { Outcome } \\
\text { Physical fitness }\end{array}$ \\
\hline Embase, Pubmed, Medline ${ }^{a}$ & $\begin{array}{l}\text { (Non-small cell lung cancer OR NSCLC } \\
\text { OR lung cancer) } \\
\text { AND } \\
\text { (patient OR geriatric OR elderly OR } \\
\text { aged OR high-risk OR vulnerable OR } \\
\text { frail) } \\
\text { NOT } \\
\text { (children OR caregiver OR tuberculosis } \\
\text { OR aneurysm) }\end{array}$ & $\begin{array}{l}\text { (prehabilitation OR before OR prior to } \\
\text { OR presurgical OR preoperative OR } \\
\text { during OR undergoing OR } \\
\text { rehabilitation OR perioperative OR } \\
\text { following OR postoperative OR lung } \\
\text { resection OR lobectomy OR physical } \\
\text { therapy OR training OR exercise OR } \\
\text { physiotherapy OR physical therapy } \\
\text { modality) } \\
\text { AND } \\
\text { (home-based OR living environment } \\
\text { OR outpatient OR home OR } \\
\text { home-based intervention) } \\
\text { NOT } \\
\text { (costs OR lung cancer screening OR } \\
\text { drug-reimbursement OR homeopathy } \\
\text { OR oxygen OR video-assisted) }\end{array}$ & $\begin{array}{l}\text { (Physical activity OR fitness OR } \\
\text { physical function OR functional status } \\
\text { OR ADL OR strength OR resistance OR } \\
\text { physical endurance) } \\
\text { NOT } \\
\text { (Genetic OR genomic OR gene OR } \\
\text { radon OR air pollution OR asbestos OR } \\
\text { cell dynamics OR drug-resistance OR } \\
\text { smoking) }\end{array}$ \\
\hline PEDro $^{a}$ & Lung cancer & Exercise $^{\mathrm{b}}$ AND home h $^{\mathrm{b}}$ & \\
\hline
\end{tabular}

${ }^{a}$ Free text words were applied to all search terms; filters were applied to language (English and Dutch included) and publication date (Jan-1-2000 until Apr-11-2016 included); search terms for population, intervention and outcome were all combined by 'AND'.

b Truncation of search terms.

$(-)$, where $\geq 6$ times 'performed' indicated high therapeutic validity. The interobserver agreement was calculated by Cohen's Kappa, with poor $(<0.20)$, reasonable $(0.21-0.40)$, moderate $(0.41-0.60)$, good $(0.61-0.80)$ or very good $(>0.80)$ agreement (Petrie and Sabin, 2009). A meta-analysis could not be performed due to clinical and statistical heterogeneity in patient samples, exercise design, study execution, and reporting of physical outcomes.

\section{Results}

The literature search identified 141 hits, leading to 107 unique studies of which 74 did not meet inclusion criteria. Thirty-three studies were selected for full-text assessment and 24 of them were excluded (conference paper with no available full-text version, $n=16)$, no physical outcome $(n=3)$, no home-based intervention $(n=2)$ or other reasons $(n=3)$. Reference tracking identified two additional articles. Eventually, 11 studies were included (Andersen et al., 2011, 2013; Arbane et al., 2011, 2014; Brocki et al., 2014; Cheville et al., 2013; Coats et al., 2013; Granger et al., 2013; Hoffman et al., 2014; Kuehr et al., 2014; Quist et al., 2012). The PRISMA flow diagram is shown in Fig. 1 (Moher et al., 2009).

\subsection{Study characteristics}

Fourhundred-fiftyone lung cancer patients were included (97\% NSCLC) with various stages of disease and treatment regimens. Ranges for sample size and mean age were 5-131 participants and 59-72 years, respectively. In Table 2, characteristics of included studies are displayed.

Only one study described prehabilitation (Coats et al., 2013) and ten studies described rehabilitation (Andersen et al., 2011, 2013; Arbane et al., 2011, 2014; Brocki et al., 2014; Cheville et al., 2013; Granger et al., 2013; Hoffman et al., 2014; Kuehr et al., 2014; Quist et al., 2012). Home-based training alone was examined in three studies (27\%) (Cheville et al., 2013; Coats et al., 2013; Hoffman et al., 2014). One study (9\%) combined home-based and extramural training (Brocki et al., 2014), six studies (55\%) combined home-based and intramural training (Andersen et al., 2011, 2013; Arbane et al., 2011, 2014; Kuehr et al., 2014; Quist et al., 2012), and one study (9\%) combined all three (Granger et al., 2013). The intervention period lasted four to 16 weeks. The home-based training component mainly consisted of resistance exercises (muscle strength) and endurance exercises (cardiorespiratory fitness)(Arbane et al., 2011; Brocki et al., 2014; Cheville et al., 2013; Coats et al., 2013; Granger et al., 2013; Hoffman et al., 2014; Kuehr et al., 2014), or mere walking (Andersen et al., 2011, 2013; Arbane et al., 2014; Quist et al., 2012). Only four interventions (36\%) were personalized (Andersen et al., 2013; Arbane et al., 2011; Granger et al., 2013; Hoffman et al., 2014). Supervision was performed by telephone calls (Arbane et al., 2014; Cheville et al., 2013; Coats et al., 2013; Granger et al., 2013; Hoffman et al., 2014; Kuehr et al., 2014), home visits (Arbane et al., 2014; Granger et al., 2013; Hoffman et al., 2014), or during intramural sessions (Andersen et al., 2011, 2013; Arbane et al., 2014; Brocki et al., 2014; Kuehr et al., 2014; Quist et al., 2012). The number of home-based training sessions varied between twice a week (Brocki et al., 2014; Cheville et al., 2013) and once a day (Andersen et al., 2013; Arbane et al., 2014; Brocki et al., 2014; Granger et al., 2013), and moderate intensity was mainly applied. Intramural and extramural training consisted of supervised endurance training combined with resistance exercises. The number of sessions varied from twice a day postoperatively (Arbane et al., 2011; Granger et al., 2013) to once a week (Brocki et al., 2014), from 10 to $30 \mathrm{~min}$ (Granger et al., 2013) up to $1.5 \mathrm{~h}$ (Andersen et al., 2011, 2013) at moderate or high intensity. These sessions took place during hospitalization after surgery followed by home-based training alone (Arbane et al., 2011, 2014; Brocki et al., 2014), intramural training combined with concurrent home-based exercises (Andersen et al., 2011; Kuehr et al., 2014; Quist et al., 2012), or intramural training with home-based exercises in between (Andersen et al., 2013; Granger et al., 2013). Five studies (45\%) included a control group that received regular care (Arbane et al., 2011, 2014; Granger et al., 2013), home-based exercises (Brocki et al., 2014), or no exercises (Cheville et al., 2013).

Physical fitness was mainly measured by cardiorespiratory fitness (6MWT distance) (Arbane et al., 2011; Brocki et al., 2014; Coats et al., 2013; Granger et al., 2013; Hoffman et al., 2014; Kuehr et al., 2014; Quist et al., 2012) and muscle strength (kilogram of resistance per muscle group) (Arbane et al., 2011, 2014; Coats et al., 2013; Kuehr et al., 2014; Quist et al., 2012). All studies (100\%) reported patient adherence (percentage) and most described reasons for drop-out. Six studies (55\%) reported on compliance of home-based exercises (percentage) (Brocki et al., 2014; Cheville et al., 2013; Granger et al., 2013; Hoffman et al., 2014; Kuehr et al., 2014; Quist et al., 2012). Treatment tolerance and recovery were 
Table 2

Description of demographics and results of included (home-based) (p)rehabilitation studies.

\begin{tabular}{|c|c|c|c|c|c|}
\hline $\begin{array}{l}\text { First author, } \\
\text { year }\end{array}$ & $\begin{array}{l}\text { Study type } \\
\text { 1. (P)rehabilitation } \\
\text { 2. Country } \\
\text { 3. Type of study } \\
\text { 4. Randomization }\end{array}$ & Demographics (IG/CG) & Intervention exercise (IG/CG) & $\begin{array}{l}\text { Intervention } \\
\text { 1. Measurement times } \\
\text { 2. Exercise time (min) } \\
\text { 3. Follow-up } \\
\text { 4. Delivery }\end{array}$ & $\begin{array}{l}\text { Outcomes (IG/CG) } \\
\text { 1. Physical fitness } \\
\text { 2. Patient adherence (drop-out) } \\
\text { Exercise adherence (compliance) } \\
\text { 3. Treatment tolerance and recovery }\end{array}$ \\
\hline Andersen, 2011 & $\begin{array}{l}\text { 1. Rehabilitation } \\
\text { 2. Denmark } \\
\text { 3. PCT } \\
\text { 4. NA }\end{array}$ & $\begin{array}{l}N=24 \\
\text { NSCLC } N=19 \\
\text { Age } \text { mean (range) } \\
\text { Men } 64 \text { (55-77) } \\
\text { Women 67 (48-76) } \\
\text { Treatment } N \\
\text { Surgery 5, CT 19, RT 8, TKI1 }\end{array}$ & $\begin{array}{l}\text { Home-based } \\
5 / \text { wk for } 7 \text { weeks unsupervised } \\
\text { diary-based aerobic } \\
\text { (walking)+ breathing } \\
\text { Intramural (simultaneously) } \\
\text { 2/wk for } 7 \text { weeks supervised } \\
\text { group sessions, aerobic } \\
(\text { walking }(85 \% \\
\left.\mathrm{VO}_{2 \max }\right) \text { + breathing } \\
\text { Control NA }\end{array}$ & $\begin{array}{l}\text { 1. T0 1st supervised training } \\
\text { T1 last supervised training } \\
\text { 2. Home training NR; } \\
\text { Intramural } 90 \\
\text { 3. Wk1-10/11 } \\
\text { 4. After surgery; during CT, RT, } \\
\text { or TKI }\end{array}$ & $\begin{array}{l}\text { 1. ISWT med diff (range) }+9 \%(-77 ; 39 \%) \\
P=0.021 \\
\text { ESWT med diff (range) }+109 \%(-70 ; 432 \%) \\
P=0.002 \\
\text { FEV }_{1} \text { med diff (range) } 0 \%(-0.3 ; 0.6 \%) P=\mathrm{NR} \\
\text { 2. Drop-out with reasons }(\mathrm{N}) \text { home-based } \\
\text { NR; intramural } \\
29 \%,<65 \% \text { of sessions present }(3), \\
\text { incomplete data ISWT ( } 4 \text { ) } \\
\text { Compliance }(N) \text { NR; continued after } \\
\text { intervention ( } 7) \\
\text { 3. NR }\end{array}$ \\
\hline Andersen, 2013 & $\begin{array}{l}\text { 1. Rehabilitation } \\
\text { 2. Denmark } \\
\text { 3. PCT } \\
\text { 4. NA }\end{array}$ & $\begin{array}{l}N=51 \\
\text { NSCLC } N=39 \\
\text { Age mean } \pm S D \\
\text { Men } 65 \pm 8, \text { women } 65 \pm 7 \\
\text { Treatment } N \\
\text { Surgery } 10, \text { CT 26, RT } 3, \text { TKI } 2\end{array}$ & $\begin{array}{l}\text { Intramural } \\
\text { 2/wk for } 3 \text { weeks aerobic } \\
\text { (cycling and walking (Borg RPE } \\
16-18) \text { )+ breathing } \\
\text { Repetition of this design after } \\
\text { home training } \\
\text { Home-based } \\
\text { 7/wk for } 3 \text { weeks (between } \\
\text { intramural sessions) } \\
\text { personalized unsupervised } \\
\text { diary instructed } \\
\text { aerobic + breathing } \\
\text { Intramural } \\
\text { 2/wk for } 3 \text { weeks intramural } \\
\text { regimen } \\
\text { Control NA }\end{array}$ & $\begin{array}{l}\text { 1. T0 } 1 \mathrm{t} \text { supervised training } \\
\text { T1 last supervised training } \\
\text { 2. Home training NR; } \\
\text { Intramural } 90 \\
\text { 3. Wk1-13 } \\
\text { 4. Unclear (after surgery); } \\
\text { during or after CT, RT, and TKI }\end{array}$ & $\begin{array}{l}\text { 1. } \mathrm{VO}_{2 \max }\left(\mathrm{ml} / \mathrm{O}_{2} / \mathrm{kg} / \mathrm{min}\right) \text { mean } \pm S D \mathrm{~T} 0 \\
14 \pm 3 \mathrm{~T} 14 \pm 3 P=0.763 \\
\mathrm{FEV} 1 \text { ( } \mathrm{L} \text { ) mean } \pm S D \mathrm{TO} 2.0 \pm 0.6 \mathrm{~T} 12.0 \pm 0.5 \\
P=\mathrm{NS} \\
\text { 2. Drop-out with reasons ( } \mathrm{N}) 43 \%, \\
\text { withdrawal after first session } \\
\text { (7), withdrawal after home sessions (9), } \\
<65 \% \text { present at } \\
\text { second session (6); After intervention, not } \\
\text { continuing physical activity (8), LFU ( } 3 \text { ) } \\
\text { Compliance ( } \mathrm{N} \text { ) NR; continued after } \\
\text { intervention (18) } \\
\text { 3. NR }\end{array}$ \\
\hline Arbane, 2011 & $\begin{array}{l}\text { 1. Rehabilitation } \\
\text { 2. UK } \\
\text { 3. RCT } \\
\text { 4. Block randomization }\end{array}$ & $\begin{array}{l}N=26 / 25 \\
\text { NSCLC } N=26 / 25 \\
\text { Stage } N \\
\text { I } 15 / 10, \text { II } 6 / 6, \text { III } 2 / 0, \text { IV 0/5, NR } \\
3 / 4 \\
\text { Age } \text { mean (range) } \\
62.6(32-47) / 65.4(47-82) \\
\text { Sex NR } \\
\text { Treatment } N \\
\text { Surgery } 26 / 25\end{array}$ & $\begin{array}{l}\text { Intramural } \\
2 / \text { day for } 5 \text { days postoperative } \\
\text { mobility + resistance (seated } \\
\text { leg raises)+ aerobic (walking, } \\
\text { cycling } 60-80 \% \mathrm{HR} \text { ) } \\
\text { Home-based } \\
12 \text { weeks personalized (based } \\
\text { on hobbies) aerobic } \\
\text { (walking)+ strength (not } \\
\text { specified) with monthly } \\
\text { supervision/home visit } \\
\text { Control } \\
\text { Monthly check-up calls and } \\
\text { usual care including } \\
\text { breathing + mobilization }\end{array}$ & $\begin{array}{l}\text { 1. T0 preoperative } \\
\text { T1 postoperative } \\
\text { day5 } \\
\text { T2 postoperative } \\
\text { wk12 } \\
\text { 2. NR } \\
\text { 3. Preoperative- } \\
\text { postoperative wk12 } \\
\text { 4. After surgery }\end{array}$ & 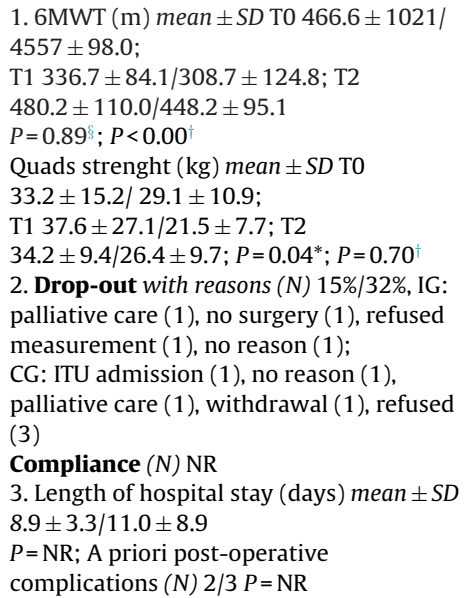 \\
\hline
\end{tabular}


Arbane, 2014

1. Rehabilitation
2. UK
3. RCT
4. Online
randomization

randomization

. Rehabilitation

2. Denmar

4. Computer-generated

randomization tables

Brocki, 2014

\section{$N=64 / 67$}

NSCLC $N=64 / 67$

Stage N I 24/ 29, II 12/12, III

$6 / 8$, IV $7 / 2$, NR $15 / 16$

Age mean $\pm S D$

$67 \pm 11 / 68 \pm 11$

Sex $N(\%)$ female

$35(55) / 24(36)$

Treatment $N$

Surgery 64/67

Comorbidities $N(\%)$

COPD 34(53)/29(43)

$=39 / 39$

NSCLC $N=39 / 36$

Stage NI 16/10, II 17/14, III 4/7,

unknown 2/15

Age mean $\pm S D$

$64 \pm 10 / 65 \pm 9$

Sex $N(\%)$ female

$19(46) / 13(35)$

Treatment $N$

Surgery $41 / 37$

COPD 8/5, DM 5/3, CVD 8/12

previous malignancy $12 / 13$

$N=26 / 33$

Lung cancer $N=16 / 18$

Stage $N$ IV 26/33

Age mean $\pm S D$

$63.8 \pm 12.5 / 65.5 \pm 8.9$

Sex $N(\%)$ female

17(51.5)/14(42.4)

Treatment $N($ NR $5 / 4)$

RT $4 / 4$, CT 24/25

\section{ntramural}

$1 /$ day for 5 days postoperative (10) $(10-\mathrm{RM}))+$ aerobic cycling (max 60\% heart rate reserve) intensity increases daily

Home-based

$7 /$ wk for 4 weeks unsupervised walking by pedometer and

weekly calls

Control

Monthly check-up calls and usual care including pain relief by breathing and mobilization exercises

\section{Extramural}

$1 /$ wk for 10 weeks starting wk3 postoperative supervised group aerobic (walking with increasing inten-

sity)+ strength + breathing Home-based

\section{(simultaneously)}

/wk strength $+7 /$ wk aerobic

walking, cycling (BORG RPE

(1-12)) diary based;

wk3-4months postoperative

Control

$2 /$ wk strength $+7 /$ wk aerobic (walking, cycling (BORG RPE

11-12)) diary based at home

\section{Home-based}

$2 /$ wk for 8 weeks strength

(Borg CR10) $+4 /$ wk for 8 weeks aerobic (walking, 1

mile/20 min) diary based and

every two weeks check-up calls

Control

No exercises or monitoring

during intervention period
1. T0 preoperative

1 postoperative

day5

2 postoperative

. Home training 30

intramural 30

3. Preoperative-postoperative

wk4

4. After surgery

T0 postoperative wk3

T1 4 months after baseline

T2 1 year after baseline

2. Home training 30

Extramural 60

3. Baseline-12 months after

baseline

4. After surgery

T0 $\mathrm{wk}$

T1 wk8

. Home training 20

. Baseline-12 month

4. During (palliative)

treatment
ISWT (m) $\operatorname{med}(\mathrm{IOR}) \mathrm{TO}$

290(180-440)/290(200-450); T1

$10(\mathrm{NR}) / 135$ (NR); T2 350(NR)/290(NR);

$P>0.05^{\ddagger} ; P>0.05$

Quads strength $(\mathrm{kg})$ mean change $\operatorname{diff}(95 \% \mathrm{BI})$ 2 4.7(0.18-0.20); $P=0.04$ in COPD patien 2. Drop-out with reasons ( $N$ ) 38\%/43\% IG:

inoperable (3), no cancer (6), ITU admission

(4), withdrawal (6), moved (1), rehospitalized

(1), deceased (1), failed activity monitor (2);

CG: inoperable (4), no cancer (1), no NSCLC (2) deceased (4), ITU admission (9), refusal (1), withdrawal (2), additional surgery (1), moved

(1), failed activity monitor (4)

Compliance $(N) \mathrm{NR}$

3. Length of hospital stay (days) mean(range) 7.5(5-8)/7.1(6-8) $P>0.05$

priori postoperative complications $(n(\%))$

20(31)/22(33) $P=\mathrm{NR}$

1. 6MWT (m) mean $\pm S D$ T0

$427 \pm 124 / 407 \pm 102$;

T1 mean diff baseline $61 \pm 52 / 55 \pm 45 P=0.57$

T2 mean diff baseline $65 \pm 70 / 60 \pm 45 P=0.93$

FEV1 (L) mean $\pm S D$ T0 $1.73 \pm 0.5 / 1.9 \pm 0.6$

11 mean diff baseline $0.14 \pm 0.3 / 0.1 \pm 0.4$

$P=0.84$

2 mean diff baseline $0.1 \pm 0.4 / 0.06 \pm 0.4$

$=0.84$

Drop-out with reasons (N) 28\%/14\% IG

deceased (3), withdrawal (8); CG: deceased (2), withdrawal (3)

Compliance Home-based (IG/CG \%) 43\%/14\%; extramural (IG N) 17 in 10 sessions, 8 in 9, 5 in 8 and 2 in 6-7 session

3. Length of hospital stay (days) mean $\pm S D$

$9 \pm 5 / 10 \pm 5 P=N R$

1. Mobility mean diff $\pm S D$ 4.88(4.66)/0.23(5.22) $P=0.002$

Activity mean diff $\pm S D 1.56 \pm 5.53 / 0.94 \pm 5.91$

$P=0.74$

teps/day (N) mean T0 3200/NR; T1 4400/NR;

$P=\mathrm{NR}$

Self-reported Ambulatory Post Acute Care Daily Activities Short Form

2. Drop-out with reasons (N) 27\%/9\% IG:

deceased (5), LFU (1),

fracture (1): CC. deceased (2) LFU (1)

Compliance (N) IG: $26,77 \%$

3. No adverse events during home-based

. 
Table 2 (Continued)

\begin{tabular}{|c|c|c|c|c|c|}
\hline $\begin{array}{l}\text { First author, } \\
\text { year }\end{array}$ & $\begin{array}{l}\text { Study type } \\
\text { 1. (P)rehabilitation } \\
\text { 2. Country } \\
\text { 3. Type of study } \\
\text { 4. Randomization }\end{array}$ & Demographics (IG/CG) & Intervention exercise (IG/CG) & $\begin{array}{l}\text { Intervention } \\
\text { 1. Measurement times } \\
\text { 2. Exercise time (min) } \\
\text { 3. Follow-up } \\
\text { 4. Delivery }\end{array}$ & $\begin{array}{l}\text { Outcomes (IG/CG) } \\
\text { 1. Physical fitness } \\
\text { 2. Patient adherence (drop-out) } \\
\text { Exercise adherence (compliance) } \\
\text { 3. Treatment tolerance and recovery }\end{array}$ \\
\hline Coats, 2013 & $\begin{array}{l}\text { 1. Prehabilitation } \\
\text { 2. Canada } \\
\text { 3. PCT } \\
\text { 4. NA }\end{array}$ & $\begin{array}{l}N=13 \\
\text { NSCLC } N=13 \\
\text { Stage } N \text { I } 5, \text { II 4, III 0, IV } 2 \\
\text { Age mean } \pm S D \text { S9 } 59 \\
\text { Sex } N \text { female } 5 \\
\text { Treatment } N \\
\text { Awaiting surgery } 10, \text { CT } 1, \text { CT } \\
\text { palliative } 1 \text {, RT + CT palliative } 1 \\
\text { Comorbidities } N \\
\text { COPD 5, hypertension 5, } \\
\text { dyslipidemia 3, DMII 2, anxiety } \\
2\end{array}$ & $\begin{array}{l}\text { Home-based } \\
3-5 / \text { wk for } 4 \text { weeks diary } \\
\text { based aerobic (cycling (Borg } \\
\text { BS } \geq 6 \text { )) + strength and weekly } \\
\text { check-up calls } \\
\text { Control NA }\end{array}$ & $\begin{array}{l}\text { 1. T0 baseline } \\
\text { T1 wk4 } \\
\text { 2. Home training } 30 \\
\text { 3. Wk1-4 } \\
\text { 4. Before treatment }\end{array}$ & 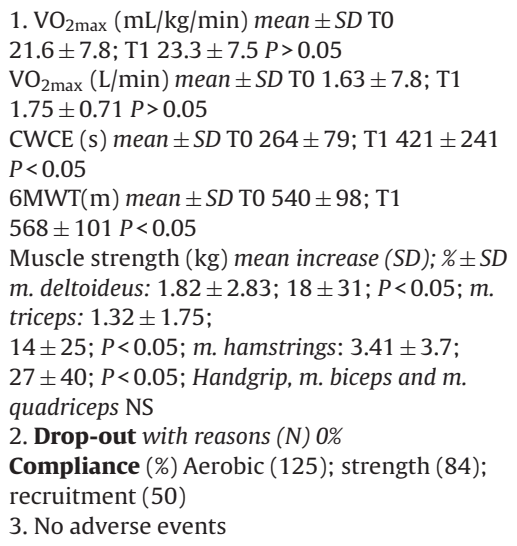 \\
\hline Granger, 2013 & $\begin{array}{l}\text { 1. Rehabilitation } \\
\text { 2. Australia } \\
\text { 3. RCT } \\
\text { 4. Computer-generated } \\
\text { randomization tables }\end{array}$ & $\begin{array}{l}N=7 / 8 \\
\text { NSCLC } N=2 / 5 \\
\text { Age } \text { mean } \pm S D \\
57 \pm 16.2 / 72.4 \pm 12.4 \\
\text { Sex \% female } \\
57.1 / 37.5 \\
\text { Treatment } N \\
\text { Surgery } 7 / 8\end{array}$ & $\begin{array}{l}\text { Intramural } \\
\text { From postoperative until } \\
\text { discharge aerobic (walking } \\
\text { (Borg BS 4) 2/day, cycling } \\
1 / \text { day)+ strength (Borg RPE 13) } \\
1 / \text { day } \\
\text { Home-based } \\
\text { 7/wk for } 2 \text { weeks after } \\
\text { discharge personalized aerobic } \\
\text { (walking (Borg BS } \\
\text { 4))+strength 1/wk check-up } \\
\text { calls and } 3 \text { home visits } \\
\text { Extramural } \\
\text { 2/wk for } 8 \text { weeks after home } \\
\text { training aerobic (walking and } \\
\text { cycling (Borg BS 4))+strength } \\
\text { (Borg RPE 13) } \\
\text { Control Standard } \\
\text { care + breathing }\end{array}$ & $\begin{array}{l}\text { 1. T0 preoperative }<2 \mathrm{wk} \\
\text { T1 postoperative } \mathrm{wk} 2 \\
\text { T2 } \text { postoperative } \\
\text { wk12 } \\
\text { 2. Home training } 30 \\
\text { Intramural } 10-30 \\
\text { Extramural } 30 \\
\text { 3. Preoperative- } \\
\text { wk12 } \\
\text { 4. After surgery }\end{array}$ & $\begin{array}{l}\text { 1. } 6 \mathrm{MWT}(\mathrm{m}) \text { mean } \pm S D \mathrm{~T} 0 \\
677.0 \pm 89.3 / 435.8 \pm 98.2 ; \\
\mathrm{T} 1647.5 \pm 53.1 / 426.0 \pm 64.3 ; \mathrm{T} 2 \\
705.7 \pm 65.3 / 458.2 \pm 38.6 \\
P=0.024 \text { at T2; others NR } \\
\text { TUG (s) mean } \pm S D \text { T0 } 6.3 \pm 1.6 / 9.0 \pm 2.6 ; \mathrm{T} 1 \\
4.4 \pm 2.6 / 6.0 \pm 3.2 ; \\
\mathrm{T} 24.9 \pm 0.8 / 6.8 \pm 1.5 P=0.041 \neq \text { at T2; others NR } \\
\text { 2. Drop-out with reasons (N) } 43 \% / 38 \% \text { IG: not } \\
\text { present for testing (1), LFU (2); CG: not present } \\
\text { for testing (2), LFU (1) } \\
\text { Compliance (\%) home-based NR; intramural } \\
\text { (71); extramural (81) } \\
\text { 3. Length of hospital stay (day) mean(range) } \\
\text { 4(3-9)/6(3-17); } P=\mathrm{NR}\end{array}$ \\
\hline Hoffman, 2014 & $\begin{array}{l}\text { 1. Rehabilitation } \\
\text { 2. United States } \\
\text { 3. PCT } \\
\text { 4. NA }\end{array}$ & $\begin{array}{l}N=5 \\
\text { NSCLC } N=5 \\
\text { Stage } N \text { IIA 1, IIB 2, IIIA } 2 \\
\text { Age mean } \pm S D \text { SD } 63.4 \pm 7.3 \\
\text { Sex } N \text { female } 3 \\
\text { Treatment } N \\
\text { Surgery+ CT } 5 \\
\text { Karnofsky PS } N \\
70 \% 2,90 \% 3 \\
\text { Comorbidities mean } N \text { (range) }\end{array}$ & $\begin{array}{l}\text { Home-based } \\
\text { 5/wk for } 16 \text { weeks from day } 4 \\
\text { post-operative personalized } \\
\text { aerobic (walking } \\
\text { ( } \leq 3 \mathrm{MET}) \text { ) + balance by } \\
\text { Nintendo Wii Fit. Two home } \\
\text { visits and weekly check-up } \\
\text { calls } \\
\text { Control NA }\end{array}$ & $\begin{array}{l}\text { 1. T0 preoperative } \\
\text { T1 postoperative wk5 } \\
\text { T2 postoperative } \\
\text { wk16 } \\
\text { 2. home-training } 5+\text { daily } 5 \\
\text { increase } \\
\text { 3. Baseline-wk16 } \\
\text { 4. After surgery and during CT }\end{array}$ & $\begin{array}{l}\text { 1. } 6 \mathrm{MWT}(\mathrm{m}) \text { mean } \pm S D \mathrm{~T} 0413 \pm 32 ; \mathrm{T} 1 \\
382 \pm 108 ; \mathrm{T} 2463 \pm 62 P=\mathrm{NR} \\
\text { Walking time (min) mean } \pm S D \mathrm{~T} 0 \mathrm{NR} ; \mathrm{T} 1 \\
24.0 \pm 4.3 ; \mathrm{T} 231.0 \pm 6.5 P=\mathrm{NR} \\
\text { 2. Drop-out } 0 \% \\
\text { Compliance } \% \pm S D(\text { range) Walking } 92.6 \pm 4.6 \\
\text { (87.0-98.1); Balance } 94.7 \pm 7.0(82.5-100) \\
\text { 3. Length of hospital stay (day) } \\
\text { mean } \pm S D(\text { range) } 8.4 \pm 2.6(5-12) P=\mathrm{NR}\end{array}$ \\
\hline
\end{tabular}




\begin{tabular}{|c|c|c|c|c|c|}
\hline Kuehr, 2014 & $\begin{array}{l}\text { 1. Rehabilitation } \\
\text { 2. Germany } \\
\text { 3. PCT } \\
\text { 4. NA }\end{array}$ & $\begin{array}{l}N=40 \\
\text { NSCLC } N=40 \\
\text { Stage } N \\
\text { IIA 2, IIIA 3, IIIB } 8, \text { IV } 27 \\
\text { Age } \text { mean } \pm S D(\text { range }) \\
60 \pm 12(22-75) \\
\text { Sex } N(\%) \text { female } 16(40) \\
\text { Treatment } N \\
\text { Surgery 3, concurrent CHRT 4, } \\
\text { sequential CHRT } 1, \text { CT } 33\end{array}$ & $\begin{array}{l}\text { Intramural } \\
5 / \text { wk for } 8 \text { weeks of which } 3 \\
\text { times supervised aerobic } \\
\text { (walking, cycling (Borg RPE } \\
\text { 12-14))+ strength (resistance } \\
\text { (Borg RPE 14-16)) } \\
\text { Home-based } \\
\text { (simultaneously) } \\
\text { 3/wk for } 8 \text { weeks diary based } \\
\text { aerobic (walking (Borg RPE } \\
\text { 12-14))+ strength (resistance } \\
\text { (Borg RPE 14-16)) and weekly } \\
\text { check-up calls } \\
\text { Control NA }\end{array}$ & $\begin{array}{l}\text { 1. T0 baseline } \\
\text { T1 wk8 } \\
\text { T2 wk16 } \\
\text { 2. Home training NR } \\
\text { Intramural NR } \\
\text { 3. Baseline-wk16 } \\
\text { 4. After surgery, during } \\
\text { CHRT and CT }\end{array}$ & $\begin{array}{l}\text { 1. } 6 \mathrm{MWT}(\mathrm{m}) \text { mean } \pm S D \text { T0 } 493 \pm 100 ; \mathrm{T} 1 \\
525 \pm 95 P<0.01 ; \mathrm{T} 2543 \pm 120 P=0.46 \\
\text { Knee flexion (Newton) mean } \pm S D \text { T0 } 140 \pm 41 ; \\
\text { T1 } 177 \pm 61 \\
P<0.01 ; \mathrm{T} 2192 \pm 57 P<0.01 \\
\text { Knee extension (Newton) mean } \pm S D \text { T0 } \\
201 \pm 86 ; \mathrm{T} 1279 \pm 71 \\
P<0.01 ; \mathrm{T} 2327 \pm 116 P<0.01 \\
\text { Other muscle strengths reported but NS } \\
\text { 2. Drop-out with reasons ( } N \text { ) } 50 \% \text { T0 fatigue (4), } \\
\text { pneumonia (1), deceased (1), pain (1), infection } \\
\text { (1), stroke (1); T1 stress (4), moved (1), } \\
\text { metastases (1), dyspnea (1), pneumonia (1), } \\
\text { stopped treatment (1); T2 no time (4), LFU (5) } \\
\text { Compliance (\%) overall ( } 82 \text { ), home-based (77), } \\
\text { intramural (95) } \\
\text { 3. NR }\end{array}$ \\
\hline Quist, 2012 & $\begin{array}{l}\text { 1. Rehabilitation } \\
\text { 2. Denmark } \\
\text { 3. PCT } \\
\text { 4. NA }\end{array}$ & $\begin{array}{l}N=29 \\
\text { NSCLC } N=19 \\
\text { Age mean(range) } \\
63(45-80) \\
\text { Sex } N(\%) \text { female 16(55.2) } \\
\text { Treatment } N \\
\text { CT } 27, \text { CHRT } 2 \\
\text { Physical activity before } \\
\text { diagnosis } N \\
\text { Sedentary }(2),<3 \mathrm{~h} / \mathrm{wk} 14, \\
\geq 3 \mathrm{~h} / \text { wk } 12,>4 \mathrm{~h} / \mathrm{wk} 1\end{array}$ & $\begin{array}{l}\text { Intramural } \\
\text { 2/wk for } 6 \text { weeks supervised } \\
\text { strength (resistance }(70-90 \% \\
\text { 1RM))+ aerobic (cycling } \\
(85-95 \% \text { max HR))+stretching } \\
\text { Home-based } \\
\text { (simultaneously) } \\
\text { 3/wk for } 6 \text { weeks unsupervised } \\
\text { diary based aerobic } \\
\text { (walking)+ relaxation } \\
\text { Control NA }\end{array}$ & $\begin{array}{l}\text { 1. T0 baseline } \\
\text { T1 wk6 } \\
\text { 2. Home training 20-60 } \\
\text { Intramural 90 } \\
\text { 3. Baseline-wk6 } \\
\text { 4. During CT and CHRT }\end{array}$ & $\begin{array}{l}1 . \mathrm{VO}_{2 \max }(\mathrm{L} / \mathrm{min}) \text { mean } \pm S D \mathrm{~T} 01.48 \pm 0.41 ; \mathrm{T} 1 \\
1.57 \pm 0.41 P=0.014 \\
6 \mathrm{MWT}(\mathrm{m}) \text { mean } \pm S D \mathrm{~T} 0524.7 \pm 88.5 ; \mathrm{T} 1 \\
564.0 \pm 88.6 P=0.006 \\
\text { FEV1 }(\mathrm{L}) \text { mean } \pm \mathrm{SD} \mathrm{T} 01.76 \pm 0.70 ; \mathrm{T} 1 \\
1.96 \pm 0.63 P=0.061 \\
\text { Muscle strength }(\mathrm{kg}) \text { mean } \pm \mathrm{SD} \text { Leg press: } \mathrm{T} 0 \\
70.34 \pm 26.9 ; \mathrm{T} 1 \text { } 86.9 \pm 28.8 \\
P=0.000 ; \text { Chest press } \mathrm{T} 030.8 \pm 13.2 ; \mathrm{T} 1 \\
40.3 \pm 16.3 \\
P=0.000 ; \text { Lat machine: } \mathrm{T} 0: 35.8 \pm 13.8 ; \mathrm{T} 1 \\
39.2 \pm 17.6 P=0.049 ; \\
\text { Abdominal crunch: } \mathrm{T} 024.9(10.7) ; \mathrm{T} 129.5(11.3) \\
P=0.000 ; \text { Lower } \\
\text { back: T0 35.3 } \pm 14.1 ; \mathrm{T} 143.1 \pm 16.2 P=0.000 ; \\
\text { Leg extension: } \\
\text { T0 38.6 } \pm 15.5 ; \mathrm{T} 145.1 \pm 18.9 P=0.000 \\
\text { 2. Drop-out with reasons }(\mathrm{N}) 21 \% \text { Loss of } \\
\text { motivation (3), worsened } \mathrm{PS}(3) \\
\text { Compliance (\%) home-based } 8.7 \% \text {, intramural } \\
\text { 73.3\% } \\
\text { 3. No adverse events }\end{array}$ \\
\hline
\end{tabular}

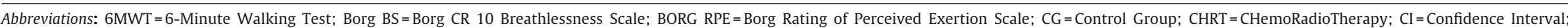

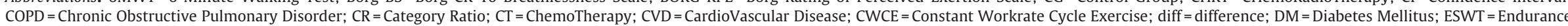

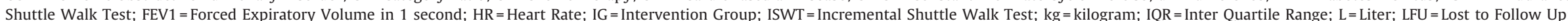

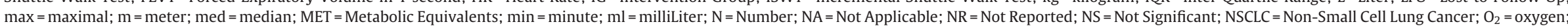

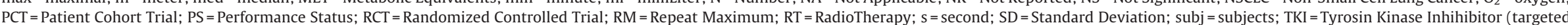
therapy); UK = United Kingdom; $\mathrm{VO}_{2 \max }=$ maximal oxygen uptake; $\mathrm{WHO}=$ World Health Organization; wk = week

Within subjects group time effect.

Between subjects group time effect.

$\S$ Between groups. 
Table 3

\begin{tabular}{lll} 
Physical outcomes including significance and clinical relevance of changes of included studies according to measuring instruments. \\
\hline Outcome unit $\quad$ First author, year $\quad$ Measurement times (IG/CG)
\end{tabular}

\begin{tabular}{|c|c|c|c|c|c|c|c|c|c|}
\hline & & & \multicolumn{3}{|c|}{ 2. Mean $\pm S D$ of change (range) $[95 \% \mathrm{CI}](\mathrm{IG} / \mathrm{CG})$} & \multirow{2}{*}{$\begin{array}{l}\text { 2. Clinically relevant } \\
\text { change }^{\mathrm{a}}\end{array}$} \\
\hline & & Baseline & Postoperative & End iv & Post iv & T0-T1 & T0-T2 & $\mathrm{T} 1-\mathrm{T} 2$ & \\
\hline \multicolumn{10}{|c|}{ Prehabilitation - cardiorespiratory fitness } \\
\hline $\mathrm{VO}_{2 \max } \mathrm{mL} / \mathrm{kg} / \min$ & Coats, 2013 & $21.6 \pm 7.8$ & & $23.3 \pm 7.5$ & & $1 .+1.7[7.8 \%]$ & & & $\begin{array}{l}\text { 1.>0.05 } \\
\text { 2. No }\end{array}$ \\
\hline $\mathrm{VO}_{2 \max } \mathrm{L} / \mathrm{min}$ & Coats, 2013 & $1.63 \pm 7.8$ & & $1.75 \pm 0.71$ & & 1. $+0.12[+7.4 \%]$ & & & $\begin{array}{l}\text { 1. }>0.05 \\
\text { 2. No }\end{array}$ \\
\hline 6MWT m & Coats, 2013 & $540 \pm 98$ & & $568 \pm 101$ & & $\begin{array}{l}1 .+28[+5.2 \%] \\
2 .+28 \pm 29\end{array}$ & & & $\begin{array}{l}\text { 1. }<\mathbf{0 . 0 5} \\
\text { 2. No }\end{array}$ \\
\hline CWCE S & Coats, 2013 & $264 \pm 79$ & & $421 \pm 241$ & & $\begin{array}{l}1 .+57[+59.5 \%] \\
2 .+157 \pm 195\end{array}$ & & & $\begin{array}{l}\text { 1. }<\mathbf{0 . 0 5} \\
\text { 2. yes }\end{array}$ \\
\hline \multicolumn{10}{|c|}{ Rehabilitation - cardiorespiratory fitness } \\
\hline $\mathrm{VO}_{2 \max } \mathrm{mL} / \mathrm{kg} / \mathrm{min}$ & Andersen, 2013 & $14 \pm 3$ & & $14 \pm 3$ & & $1 .+0.0[+0.0 \%]$ & & & $\begin{array}{l}\text { 1. } 0.763 \\
\text { 2. No }\end{array}$ \\
\hline $\mathrm{VO}_{2 \max } \mathrm{L} / \mathrm{min}$ & Quist, 2012 & $1.48 \pm 0.41$ & & $1.57 \pm 0.41$ & & $\begin{array}{l}1 .+0.09[+6.1 \%] \\
2 .+0.09[0.02 ; 0.16]\end{array}$ & & & $\begin{array}{l}\text { 1. } 0.014 \\
\text { 2. NR }\end{array}$ \\
\hline $\mathrm{FEV}_{1} \mathrm{~L}$ & Andersen, 2013 & $2.0 \pm 0.6$ & & $2.0 \pm 0.5$ & & $1 .+0.0[+0.0 \%]$ & & & $\begin{array}{l}\text { 1. NS } \\
\text { 2. No }\end{array}$ \\
\hline $\mathrm{FEV}_{1} \mathrm{~L}$ & Brocki, 2014 & $1.73 \pm 0.5 / 1.9 \pm 0.6$ & & $\begin{array}{l}\text { Mean diff } \\
0.14 \pm 0.3 / 0.1 \pm 0.4\end{array}$ & $\begin{array}{l}\text { Mean diff } \\
0.1 \pm 0.4 / 0.06 \pm 0.4\end{array}$ & $\begin{array}{l}1 .+0.14 \mid+0.1 \\
{[+8.1 /+5.3 \%]} \\
\text { 2. } 0.02[-0.14 ; 0.17]^{8}\end{array}$ & $\begin{array}{l}1 .+0.1 /+0.06 \\
{[+5.8 \mid+3.2 \%]} \\
2.0 .02[-0.14 ; 0.18]^{8}\end{array}$ & 1. $-0.04 /-0.04$ & $\begin{array}{l}\text { 1. T1 } 0.84 \\
\text { T2 } 0.84 \\
\text { 2. No }\end{array}$ \\
\hline FEV1 L & Quist, 2012 & $1.76 \pm 0.7$ & & $1.96 \pm 0.6$ & & $\begin{array}{l}1 .+0.20[+11.4 \%] \\
2.0 .20[-0.01 ; 0.41]\end{array}$ & & & $\begin{array}{l}\text { 1. } 0.061 \\
\text { 2. No }\end{array}$ \\
\hline 6MWT m & Arbane, 2011 & $\begin{array}{l}466.6 \pm 102.1 / \\
455.7 \pm 98.0\end{array}$ & $\begin{array}{l}\mathrm{D} 5336.7 \pm 84.1 / \\
308.7 \pm 124.8\end{array}$ & $\begin{array}{l}480.2 \pm 110.0 / \\
448.2 \pm 95.1\end{array}$ & & $\begin{array}{l}\text { 1. }-129.9 /-147 \\
{[-27.8 /-32.3 \%]}\end{array}$ & $\begin{array}{l}1 .+13.6 /-7.5 \\
{[+2.9 /-1.6 \%]}\end{array}$ & $\begin{array}{l}1 .+143.5 /+139.5 \\
{[+42.6 /+45.2 \%]}\end{array}$ & $\begin{array}{l}\text { 1. }<\mathbf{0 . 0 0 1} ; 0.89 \\
\text { 2. Yes }\end{array}$ \\
\hline 6MWT m & Brocki, 2014 & $427 \pm 124 / 407 \pm 102$ & & $\begin{array}{l}\text { Mean diff } \\
+61 \pm 52 /+55 \pm 45\end{array}$ & $\begin{array}{l}\text { Mean diff } \\
+65 \pm 70 /+60 \pm 45\end{array}$ & $\begin{array}{l}1 .+61 \pm 52 \mid+55 \pm 45 \\
{[+14.3 /+13.5 \%]} \\
2.8 .33[-20 ; 36.27]\end{array}$ & $\begin{array}{l}1 .+65 \pm 70 /+60 \pm 45 \\
{[+15.2 \mid+14.7 \%]} \\
2.1 .31[-28.18 ; 30.8]\end{array}$ & 1. $+4.0 /+5.0$ & $\begin{array}{l}\text { 1. T1 } 0.57^{8} \\
\text { T2 } 0.93^{8} \\
\text { 2. Yes }\end{array}$ \\
\hline 6MWT m & Granger, 2013 & $\begin{array}{l}677.0 \pm 89.3 / \\
435.8 \pm 98.2\end{array}$ & $\frac{\mathrm{Wk2} 647.5 \pm 53.1 /}{426.0 \pm 64.3}$ & $\begin{array}{l}705.7 \pm 65.3 / \\
458.2 \pm 38.6\end{array}$ & & $\begin{array}{l}\text { 1. }-29.5 /-9.8 \\
{[-4.4 /-2.3 \%]}\end{array}$ & $\begin{array}{l}1 .+28.7 \mid+22.4 \\
{[+4.2 \mid+5.1 \%]}\end{array}$ & $\begin{array}{l}1 .+58.2 /+32.2 \\
{[+9.0 /+7.6 \%]}\end{array}$ & $\begin{array}{l}\text { 1. T2 } \mathbf{0 . 0 2 4} \\
\text { 2. Yes }\end{array}$ \\
\hline 6MWT m & Hoffman, 2014 & $\begin{array}{l}413 \pm 32 \\
(367-452)\end{array}$ & $\frac{\mathrm{Wk5} 382 \pm 108}{(202-480)}$ & $\begin{array}{l}463 \pm 62 \\
(383-529)\end{array}$ & & 1. $-31[-7.5 \%]$ & 1. $+50[+12.1 \%]$ & 1. $+81[+21.2 \%]$ & $\begin{array}{l}\text { 1. NR } \\
\text { 2. Yes }\end{array}$ \\
\hline 6MWT m & Kuehr, 2014 & $493 \pm 100$ & & $525 \pm 95$ & $\begin{array}{l}\text { Wk } 16 \\
543 \pm 120\end{array}$ & $1 .+32[+6.5 \%]$ & 1. $+50[+10.1 \%]$ & 1. $+18[+3.4 \%]$ & $\begin{array}{l}\text { 1. T0-T1< }<\mathbf{0 . 0 1} \\
\text { T0-T2 } 0.46 \\
\text { 2. Yes }\end{array}$ \\
\hline 6MWT m & Quist, 2012 & $524.7 \pm 88.5$ & & $564.0 \pm 88.6$ & & $\begin{array}{l}1 .+39.3[+7.6 \%] \\
2 .+39.3[12.5 ; 66.1]\end{array}$ & & & $\begin{array}{l}\text { 1. } \mathbf{0 . 0 0 6} \\
\text { 2. No }\end{array}$ \\
\hline ISWT m & Andersen, 2011 & $\mathrm{NR}$ & & $\begin{array}{l}\text { Med diff\% } \\
+9.0(-77 ; 39)\end{array}$ & & $2 .+9.0 \%(-77 ; 39)$ & & & $\begin{array}{l}\text { 1. } \mathbf{0 . 0 2 1} \\
\text { 2. NR }\end{array}$ \\
\hline ISWT m & Arbane, 2014 & $\begin{array}{l}290(180-440) / \\
290(200-450)\end{array}$ & D5 110/135 & $350 / 290$ & & $\begin{array}{l}\text { 1. }-180 /-155 \\
{[-62 /-53 \%]}\end{array}$ & 1. $+60 /+0[+21 /+0 \%]$ & $\begin{array}{l}1 .+250 /+155 \\
{[+227 /+115 \%]}\end{array}$ & $\begin{array}{l}\text { 1. }>0.055^{\prime}>0.05 \| \\
\text { 2. Yes }\end{array}$ \\
\hline ESWT m & Andersen, 2011 & $\mathrm{NR}$ & & $\begin{array}{l}\text { Med diff } \% \\
+9(-70 ; 432)\end{array}$ & & 2. $+9 \%(-70 ; 432)$ & & & $\begin{array}{l}\text { 1. } \mathbf{0 . 0 0 2} \\
\text { 2. NR }\end{array}$ \\
\hline Steps/day n & Cheville, 2013 & 3200 & & 4400 & & $1 .+1200[+37.5 \%]$ & & & $\begin{array}{l}\text { 1. NR } \\
\text { 2. NA }\end{array}$ \\
\hline Walking time min & Hoffman, 2014 & NR & $\underline{\text { Wk }} \underline{5} 24.0 \pm 4.3$ & $31.0 \pm 6.5$ & & $1 .+7.0[+29 \%]$ & & & $\begin{array}{l}\text { 1. NR } \\
\text { 2. NA }\end{array}$ \\
\hline TUG $s$ & Granger, 2013 & $6.3 \pm 1.6 / 9.0 \pm 2.6$ & $\frac{\mathrm{Wk2}}{6.0 \pm 3.4 \pm 2.6}$ & $4.9 \pm 0.8 / 6.8 \pm 1.5$ & & $\begin{array}{l}\text { 1. }-1.9 /-3.0 \\
{[-30.2 /-30 \%]}\end{array}$ & $\begin{array}{l}\text { 1. }-1.4 \mid-2.2 \\
{[-22.2 /-24.4 \%]}\end{array}$ & $\begin{array}{l}1 .+0.5 /+0.8 \\
{[+11.4 /+13.3 \%]}\end{array}$ & $\begin{array}{l}\text { 1. } \mathbf{0 . 0 4 1} \\
\text { 2. No }\end{array}$ \\
\hline Mobility likert scale & Cheville, 2013 & $\mathrm{NR} / \mathrm{NR}$ & & NR/NR & & $\begin{array}{l}2 .+4.88 \pm 4.66[2.96 ; \\
-6.80] / \\
+0.23 \pm 5.22[-1.76 ; 2.22]\end{array}$ & & $\begin{array}{l}\text { 1. } .002 \\
\text { 2. NA }\end{array}$ & \\
\hline Activity likert scale & Cheville, 2013 & NR/NR & & NR/NR & & $\begin{array}{l}2 . \\
+1.56 \pm 5.53[-0.72 ; 3.82] \\
\mid+0.94 \pm 5.91[-1.26 ; 3.14\end{array}$ & & $\begin{array}{l}\text { 1. } 0.74 \\
\text { 2. NA }\end{array}$ & \\
\hline
\end{tabular}




\begin{tabular}{|c|c|c|c|c|c|c|c|c|c|}
\hline \multicolumn{10}{|c|}{ Prehabilitation - muscle strength } \\
\hline m. deltoi-deus kg & Coats 2013 & \multicolumn{2}{|l|}{ NR } & \multicolumn{2}{|l|}{ NR } & \multicolumn{3}{|l|}{$\begin{array}{l}1 .+1.82 \pm 2.83 \\
{[+18.0 \% \pm 31.0]}\end{array}$} & $\begin{array}{l}\text { 1. }<\mathbf{0 . 0 5} \\
\text { 2. NA }\end{array}$ \\
\hline m. triceps kg & Coats 2013 & \multicolumn{2}{|c|}{$\mathrm{NR}$} & \multicolumn{2}{|l|}{ NR } & \multicolumn{3}{|l|}{$1 .+1.32 \pm 1.75$} & $1 .<0.05$ \\
\hline m. ham-strings kg & Coats 2013 & \multicolumn{2}{|l|}{ NR } & \multicolumn{2}{|l|}{ NR } & \multicolumn{3}{|l|}{$\begin{array}{l}1 .+3.41 \pm 3.7 \\
{[+27.0 \% \pm 40.0]}\end{array}$} & $\begin{array}{l}\text { 2. NA } \\
\text { 1. }<0.05 \\
\text { 2. NA }\end{array}$ \\
\hline \multicolumn{10}{|c|}{ Rehabilitation - muscle strength } \\
\hline m. quadri-ceps kg & Arbane 2011 & $\begin{array}{l}33.2 \pm 15.2 / \\
29.1 \pm 10.9\end{array}$ & \multirow[t]{2}{*}{$\frac{\mathrm{D} 5}{21.5} 3 \pm .6 \pm 27.7$} & \multicolumn{2}{|c|}{$34.2 \pm 9.4 / 26.4 \pm 9.7$} & $\begin{array}{l}1 .+4.4 /-7.6 \\
{[+13.3 /-26.1]}\end{array}$ & $\begin{array}{l}1 .+1.0 /-2.7 \\
{[+3.0 /-9.3 \%]}\end{array}$ & $\begin{array}{l}1 .-3.4 /+4.9 \\
{[-9.0 /+22.8 \%]}\end{array}$ & $\begin{array}{l}\text { 1. } 0.70^{\|} ; \mathbf{0 . 0 4} \\
\text { 2. NA }\end{array}$ \\
\hline Extension knee $\mathrm{N}$ & Kuehr 2014 & $201 \pm 86$ & & $279 \pm 71$ & $\begin{array}{l}\text { Wk16 } \\
327 \pm 116\end{array}$ & $1 .+78[+38.8 \%]$ & $1 .+126[+62.7 \%]$ & $1 .+48[+17.2 \%]$ & $\begin{array}{l}\text { 1. T0-T1 }<\mathbf{0 . 0 1} \\
\text { T0-T2 }<\mathbf{0 . 0 1} \\
\text { 2. NA }\end{array}$ \\
\hline Extension leg kg & Quist et 2012 & $38.6 \pm 15.5$ & & $45.1 \pm 18.9$ & & $1 .+6.5[+16.8 \%]$ & & & 1.0 .000 \\
\hline & & & & & & 2. $6.5[4.1-8.9]$ & & & 2. NA \\
\hline Press leg $\mathrm{kg}$ & Quist 2012 & $70.34 \pm 26.9$ & & $86.9 \pm 28.8$ & & $1 .+16.56[+23.5 \%]$ & & & 1.0 .000 \\
\hline & & & & & & 2. $+16.5[11.5-21.7$ & & & 2. NA \\
\hline Extension elbow $\mathrm{N}$ & Kuehr 2014 & $124 \pm 44$ & & $136 \pm 44$ & $\begin{array}{l}\text { Wk16 } \\
129 \pm 41\end{array}$ & 1. $+12[+9.7 \%]$ & $1 .+5[+4.0 \%]$ & 1. $-7[-5.1 \%]$ & $\begin{array}{l}\text { 1. T0-T1 }<\mathbf{0 . 0 1} \\
\text { T0-T2 } 0.49 \text { 2. NA }\end{array}$ \\
\hline Flexion knee $\mathrm{N}$ & Kuehr 2014 & $140 \pm 41$ & - & $177 \pm 61$ & $\begin{array}{l}\text { Wk16 } \\
192 \pm 57\end{array}$ & $1 .+33[+23.6 \%]$ & $1 .+52[+37.1 \%]$ & $1 .+15[+8.5 \%]$ & $\begin{array}{l}\text { 1. T0-T1 }<\mathbf{0 . 0 1} \\
\text { TO-T2 }<\mathbf{0 . 0 1}\end{array}$ \\
\hline Flexion elbow $\mathrm{N}$ & Kuehr 2014 & $144 \pm 52$ & - & $152 \pm 55$ & $\begin{array}{l}\text { Wk16 } \\
158 \pm 69\end{array}$ & $1 .+8[+5.6 \%]$ & 1. $+14[+9.7 \%]$ & $1 .+6[+3.9 \%]$ & $\begin{array}{l}\text { 2. NA } \\
\text { 1. T0-T1 0.02 } \\
\text { T0-T2 } 0.68 \\
\text { 2. NA }\end{array}$ \\
\hline Hip flexion $\mathrm{N}$ & Kuehr 2014 & $133 \pm 48$ & - & $137 \pm 54$ & $\begin{array}{l}\text { Wk16 } \\
135 \pm 62\end{array}$ & 1. $+5[+3.8 \%]$ & $1 .+2[+1.5 \%]$ & 1. $-2[-1.5 \%]$ & $\begin{array}{l}\text { 1. T0-T1 } 0.21 \\
\text { T0-T2 } 0.26 \\
\text { 2. NA }\end{array}$ \\
\hline Hip abduction N & Kuehr 2014 & $153 \pm 45$ & - & $164 \pm 48$ & $\begin{array}{l}\text { Wk16 } \\
161 \pm 48\end{array}$ & $1 .+11[+7.2 \%]$ & 1. $+8[+5.2 \%]$ & 1. $-3[-1.8 \%]$ & $\begin{array}{l}\text { 1. T0-T1 }<\mathbf{0 . 0 1} \\
\text { TO-T2 } 0.73 \\
\text { 2. NA }\end{array}$ \\
\hline Chest press $\mathrm{kg}$ & Quist 2012 & $30.8 \pm 13.2$ & - & $40.4 \pm 16.3$ & - & $\begin{array}{l}1 .+9.6[+31.2 \%] \\
2+9.5[6.4-127]\end{array}$ & - & - & 1.0 .000 \\
\hline Lat ma-chine $\mathrm{kg}$ & Quist 2012 & $35.8 \pm 13.8$ & - & $39.2 \pm 17.6$ & - & $1 .+3.4[+9.5 \%]$ & - & - & 1. 0.049 \\
\hline Abdominal crunch $\mathrm{kg}$ & Quist 2012 & $24.9 \pm 10.7$ & - & $29.5 \pm 11.3$ & - & $\begin{array}{l}2 .+3.4[0.0-6.7] \\
1 .+4.6[+18.5 \%]\end{array}$ & - & - & $\begin{array}{l}\text { 2. NA } \\
\text { 1. } 0.000\end{array}$ \\
\hline & & & & & & $2 .+4.6[3.2-6.0]$ & & & 2. NA \\
\hline Lower back kg & Quist 2012 & $35.3 \pm 14.1$ & - & $43.1 \pm 16.2$ & - & $\begin{array}{l}1 .+7.8[+22.1 \%] \\
2 .+7.8[4.8-10.8]\end{array}$ & - & - & $\begin{array}{l}\text { 1. } 0.000 \\
\text { 2. NA }\end{array}$ \\
\hline
\end{tabular}

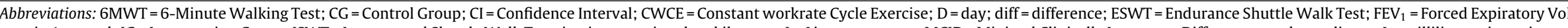

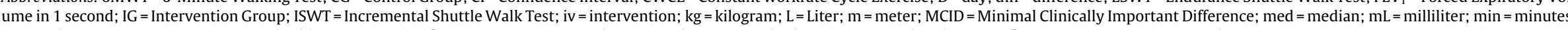

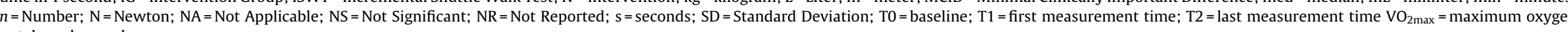

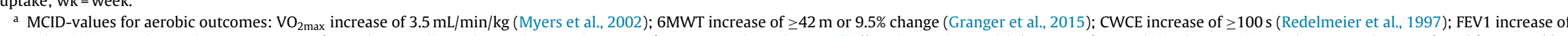

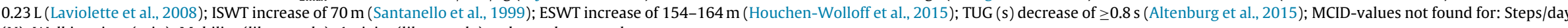
(N); Walking time (min); Mobility (likert scale); Activity (likert scale) and muscle strengths.

Within subjects group time effect.

Between subjects group time effect.

$\S$ Between groups.

Between measurement times. 


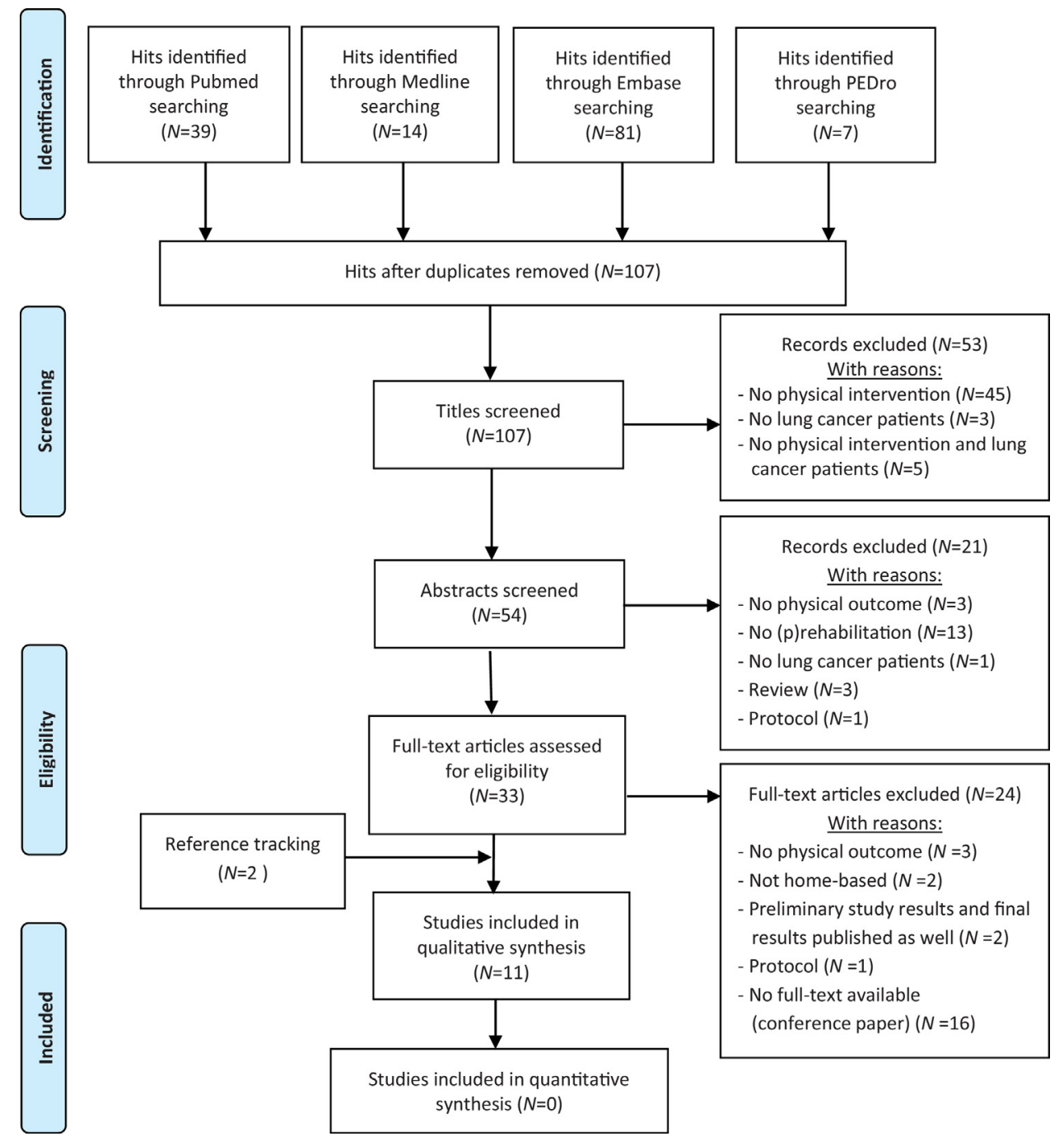

Fig. 1. PRISMA flow diagram displaying the selection of studies and reasons for exclusion.

described as adverse events (including post-operative pulmonary complications) (Cheville et al., 2013; Coats et al., 2013), or hospitalization days (Arbane et al., 2011, 2014; Brocki et al., 2014; Granger et al., 2013; Hoffman et al., 2014). Other results of physical outcomes are displayed according to measuring instruments in Table 3 (Myers et al., 2002; Redelmeier et al., 1997; Laviolette et al., 2008; Santanello et al., 1999; Houchen-Wolloff et al., 2015; Altenburg et al., 2015; Wright et al., 2011).

\subsection{Results of individual studies}

The prehabilitation study and six rehabilitation studies (64\%) described significantly improved physical fitness after (homebased) training (Andersen et al., 2011; Arbane et al., 2011; Coats et al., 2013; Granger et al., 2013; Kuehr et al., 2014; Quist et al., 2012). Three additional rehabilitation studies (27\%) indicated improved physical fitness as well, although significance was not reported (Brocki et al., 2014; Cheville et al., 2013; Hoffman et al., 2014). Specific outcomes are displayed in Table 2. The 6MWT distance was described in seven studies (64\%), including the prehabilitation study, and showed a significant improvement of 28-65 m (5.2-43\%) (Arbane et al., 2011; Brocki et al., 2014; Coats et al., 2013; Granger et al., 2013; Hoffman et al., 2014; Kuehr et al., 2014; Quist et al., 2012). In five of them, this gain was clinically relevant (Arbane et al., 2011; Brocki et al., 2014; Granger et al., 2013; Hoffman et al.,
2014; Kuehr et al., 2014). Muscle strength increased significantly during training sessions for most muscle groups (Arbane et al., 2014; Coats et al., 2013; Kuehr et al., 2014; Quist et al., 2012). All studies described patient adherence and varied from 50\% (Kuehr et al., 2014) to $100 \%$ (Coats et al., 2013; Hoffman et al., 2014). Main reasons for dropout were clinical deterioration, incomplete dataassessment, dying, and withdrawal from study-protocol. In all three studies examining home-based training alone, patient adherence was sufficient ( $72 \%, 100 \%$, and $100 \%$, respectively) (Cheville et al., 2013; Coats et al., 2013; Hoffman et al., 2014). In three of eight studies combining home-based with extramural and/or intramural training, patient adherence was sufficient $(71 \%, 72 \%$, and $79 \%$, respectively) (Andersen et al., 2011; Brocki et al., 2014; Quist et al., 2012), and varied from 50 to $68 \%$ in the remaining five studies (Andersen et al., 2013; Arbane et al., 2011, 2014; Granger et al., 2013; Kuehr et al., 2014). Reported exercise adherence for the home-based component varied from 9\% (Quist et al., 2012) to 125\% (Coats et al., 2013) and was sufficient in four of six studies (Cheville et al., 2013; Coats et al., 2013; Hoffman et al., 2014; Kuehr et al., 2014). Six studies included adverse events or hospitalization time (55\%). For surgical patients, hospitalization varied in the intervention group from four (Granger et al., 2013) to nine days (Brocki et al., 2014), compared to six (Granger et al., 2013) to eleven days (Arbane et al., 2011) in the control group. Adverse events were absent (Coats 
Table 4

Results of methodological quality according to 'the Cochrane risk of bias tool'.

\begin{tabular}{|c|c|c|c|c|c|c|c|}
\hline First author year & $\begin{array}{l}\text { Randomization } \\
\text { (selection bias) }\end{array}$ & $\begin{array}{l}\text { Equal groups } \\
\text { (selection bias) }\end{array}$ & $\begin{array}{l}\text { Blinding of } \\
\text { participants and } \\
\text { personnel } \\
\text { (performance } \\
\text { bias) }\end{array}$ & $\begin{array}{l}\text { Blinding of } \\
\text { outcome } \\
\text { assessment } \\
\text { (detection bias) }\end{array}$ & $\begin{array}{l}\text { Selective } \\
\text { drop-out } \\
\text { (attrition bias) }\end{array}$ & $\begin{array}{l}\text { Selective } \\
\text { reporting } \\
\text { (reporting bias) }\end{array}$ & $\begin{array}{l}\text { Methodological } \\
\text { quality }\end{array}$ \\
\hline Andersen 2011 & + & NA & NA & NA & - & - & Mode-rate \\
\hline Andersen 2013 & + & NA & NA & NA & + & - & Low \\
\hline Arbane 2011 & - & - & + & - & + & + & Low \\
\hline Arbane 2014 & + & - & + & + & + & + & Low \\
\hline Brocki 2014 & - & - & + & - & - & - & High \\
\hline Cheville 2013 & + & - & + & + & - & + & Low \\
\hline Coats 2013 & + & NA & NA & NA & - & - & Mode-rate \\
\hline Granger 2013 & - & + & + & - & + & + & Low \\
\hline Hoffman 2014 & + & NA & NA & NA & - & - & Mode-rate \\
\hline Kuehr 2014 & + & NA & NA & NA & + & - & Low \\
\hline Quist 2012 & + & NA & NA & NA & + & + & Low \\
\hline
\end{tabular}

Risk of bias: + 'present', - 'absent'; Methodological quality: present bias $\leq 17 \%$ 'high', 18-33\% 'moderate', $\geq 50 \%$ 'low'; NA 'not applicable'.

et al., 2013; Quist et al., 2012) or occurred infrequently during the postoperative period (Arbane et al., 2011).

\subsection{Qualitative assessment}

Only one study (9\%) had a high methodological quality and was at risk for performance bias as participants nor personnel were blinded (Table 4; Cochrane Statistical Methods Group and Cochrane Bias Methods Group, 2011a; Brocki et al., 2014). Therapeutic validity was low in this study due to insufficient eligibility criteria for therapist and setting, rationale for the training program, and monitoring or personalization of the intervention (Table 5; Hoogeboom et al., 2012). A moderate methodological quality was found in two rehabilitation studies and one prehabilitation study (45\%), where especially selection bias could influence study outcomes (Andersen et al., 2011; Coats et al., 2013; Hoffman et al., 2014). Additionally, therapeutic validity was high in all studies. In the seven remaining rehabilitation studies, a low methodological quality was found due to selection (Andersen et al., 2013; Arbane et al., 2014; Cheville et al., 2013; Granger et al., 2013; Kuehr et al., 2014; Quist et al., 2012), reporting (Arbane et al., 2011, 2014; Cheville et al., 2013; Granger et al., 2013; Quist et al., 2012), performance (Arbane et al., 2011, 2014; Brocki et al., 2014; Cheville et al., 2013; Granger et al., 2013), and attrition bias (Andersen et al., 2013; Arbane et al., 2011, 2014; Granger et al., 2013; Kuehr et al., 2014; Quist et al., 2012). Of these seven studies, three had a low therapeutic validity due to lack of eligibility criteria (Arbane et al., 2011, 2014; Kuehr et al., 2014; Quist et al., 2012), lack of rationale for the content and intensity of the program (Arbane et al., 2011; Quist et al., 2012), or no personalized exercises (Arbane et al., 2014; Kuehr et al., 2014; Quist et al., 2012). The three studies assessing home-based training alone had a high therapeutic validity, and a moderate (Coats et al., 2013; Hoffman et al., 2014), and low (Cheville et al., 2013) methodological quality. The interobsever agreement for methodological quality was very good (kappa 0.80) and for therapeutic validity good (kappa $0.76)$.

Across included studies, only one described specific demands for therapist and setting (Hoffman et al., 2014) and four did personalize exercises (Andersen et al., 2013; Arbane et al., 2014; Granger et al., 2013; Hoffman et al., 2014). Selective dropout in six studies could have led to attrition bias (Andersen et al., 2013; Arbane et al., 2011, 2014; Granger et al., 2013; Kuehr et al., 2014; Quist et al., 2012). Five studies did not display results entirely or did not report on significance, thereby inducing reporting bias (Arbane et al., 2011, 2014; Cheville et al., 2013; Granger et al., 2013; Quist et al., 2012). The included randomized controlled trials did not perform blinding for randomization, participants, or personnel. Therefore, these studies were at risk for performance and detection bias (Arbane et al., 2011, 2014; Brocki et al., 2014; Cheville et al., 2013; Granger et al., 2013).

\section{Discussion}

The aim of this systematic review was to evaluate feasibility and effectiveness of prehabilitation and rehabilitation including a home-based component for patients with NSCLC and to describe physical fitness, adherence, treatment tolerance, and recovery.

\subsection{Summary of evidence}

Ten rehabilitation studies and one prehabilitation study were included (451 patients with NSCLC). Patients included in this review were diagnosed with stage I-IV disease, and most underwent surgery with or without (neo)adjuvant treatment (308 of 496 (62\%)). However, studies in which patients received radiotherapy, chemotherapy, chemoradiotherapy, or palliative care were also included. Rehabilitation studies including a home-based component showed significantly or clinically relevant increased physical fitness. Home-based prehabilitation may increase physical fitness as well, although only one published study was found. Patient adherence and exercise adherence were generally higher and sufficient in home-based interventions compared to combinations of home-based, intramural- and/or extramural training. It remains unclear wether home-based prehabilitation can lead to less adverse events or hospitalization time, while rehabilitation including a home-based component might improve recovery after treatment.

Highest patient adherence and/or exercise adherence together with significant or clinically relevant improvements in physical fitness can be reached by home-based interventions, as was seen in three studies including home-based interventions only (Cheville et al., 2013; Coats et al., 2013; Hoffman et al., 2014). However, it should be mentioned that these were two pilot studies (Coats et al., 2013; Hoffman et al., 2014) and one randomized controlled trial (Cheville et al., 2013), and other included studies with a homebased component did not always report compliance of home-based exercises (Andersen et al., 2011, 2013; Arbane et al., 2011, 2014; Granger et al., 2013). Still, physical fitness did not differ between home-based training alone and those with additional extramural sessions (Brocki et al., 2014). Probably, newly diagnosed lung cancer patients show interest and motivation for exercise programs, in which home-based training during treatment was preferred (Karvinen et al., 2016). As patients with NSCLC are often older and frail (Hsu et al., 2015; Semrau et al., 2014; Hoogeboom et al., 2012), commuting and accessibility to in- and outpatient facilities hin- 


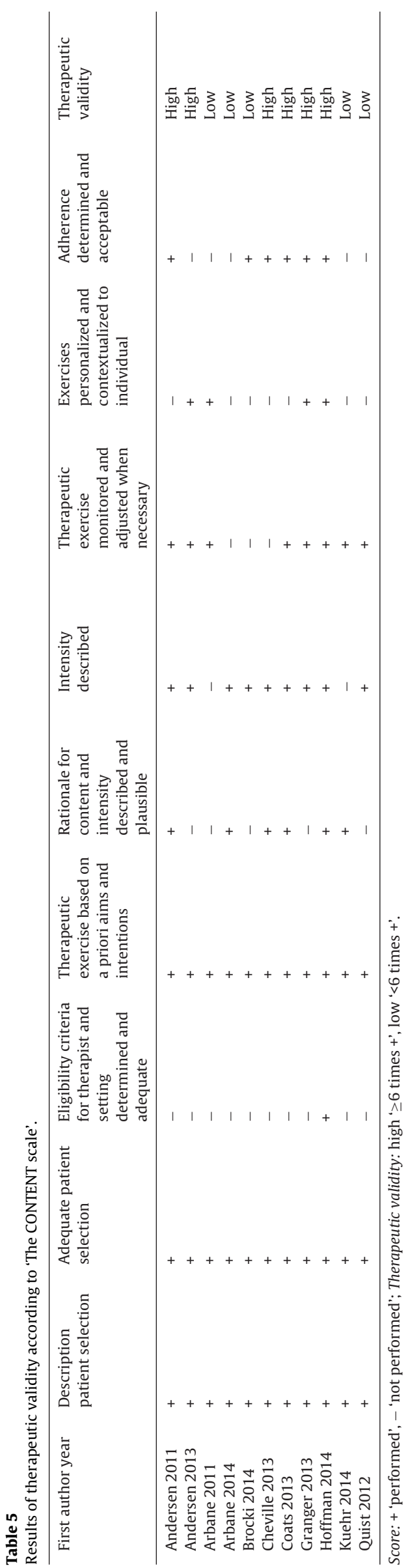

der participation in clinic-based exercise programs (Oosting et al., 2012). Therefore, exercise programs should be delivered at the patient's own living situation to optimize adherence (Oosting et al., 2012). Furthermore, regular supervision (Dalal et al., 2010), and a personalized training program (Spruit et al., 2013; Royal Dutch Physiotherapy Society, 2008; Netherlands Cancer Registry, 2011; Pouwels et al., 2015) can further increase adherence rates by facilitating motivation and a physically active lifestyle, as is recognized by the CONTENT scale (Hoogeboom et al., 2012; Herbert and Bø, 2005). At the time of cancer diagnosis, patients are more susceptible to persue a healthy lifestyle to optimize treatment outcomes and general health (Westmaas et al., 2015). In two included studies, increased physical fitness maintained several weeks (Kuehr et al., 2014), and ten months (Brocki et al., 2014) after the intervention without additional exercise instructions. This was previously found in surgical patients with NSCLC receiving prehabilitation and/or rehabilitation ((p)rehabilitation), although home-based exercises were not included in these studies (Ni et al., 2016; Cavalheri et al., 2014). Despite the fact that improvement of treatment tolerance or recovery is the driving force behind the concept of (p)rehabilitation, it was either not reported or reported by diverse parameters (number of complications or hospitalization days). Trials mainly included fit and selected patients without comorbidities, leaving older and high-risk patients underrepresented (Kilari et al., 2016).

Most rehabilitation studies combine intramural and/or extramural training with home-based exercises. Also, there is heterogeneity in the contents of training sessions, and their planning and sequences. Higher training intensity and use of devices such as bicycles and treadmills were more present in intramural and extramural sessions, whereas home-based sessions included lower intensity and more simple instruments. The broad patient population provided in our review included different stages of disease, treatment options, age groups, and physical fitness. This heterogeneity reflects everyday clinical care and probably explains the observed wide range of effect sizes and maximal capable improvements in treatment outcomes. However, it hinders the interpretation of summarized results, and effects of interactions between training contents and patient characteristics are warranted. Furthermore, natural physical recovery comes into play as increased physical fitness cannot be explained by the intervention solely, emphasizing the importance of control groups in studies.

\subsection{Strengths and limitations}

Strengths of this study are the independent literature search, selection, and data extraction by two reviewers with good agreement, thereby preventing errors in study and data selection, and limiting reporting bias (Cochrane Statistical Methods Group and Cochrane Bias Methods Group, 2011b). Also, therapeutic validity was assessed, providing more insight in reported study outcomes, as a low therapeutic quality might explain decreased effectiveness of the intervention compared to what was expected (Hoogeboom et al., 2012). Nevertheless, the outcomes of this systematic review should be interpreted carefully due to several contstraints. Only three studies incorporated home-based training alone for (p)rehabilitation. The eight remaining studies included home-based, intramural- and/or extramural components and as a result, seperate effects of a home-based training component could not be attributed. Also, several pilot studies were included. This means that evidence is still lacking and powered randomized controlled trials are required. Especially for home-based prehabilitation, not all potential eligible studies were available. As negative and non-significant outcomes are less likely to be published, publication bias could lead to a more positive scope of outcomes. Regarding methodological quality, cut off values were not present and categories were arbitrarily chosen. This could lead to an over- 
estimation of methodological quality as some types of bias are determined by more than one study characteristic (The Cochrane Collaboration, 2011). Moreover, some components did apply to randomized controlled trials only. Since clinical and statistical heterogeneity impeded the interpretation of patient characteristics and exercise contents, the internal validity of summarized effect sizes for the 6MWT distance is questionable. Therefore, a meta-analysis could not be performed despite the vast number of studies and patients included (Herbert and Bø, 2005). Furthermore, the goal of (p)rehabilitation will be different for patients receiving surgery, radiotherapy, chemoradiotherapy, chemotherapy, and palliative treatment. As the included patient group was heterogeneous with different prognoses and applied treatment regimens, various effects can be expected which cannot be explained by (lack of) effectiveness of ( $p$ )rehabilitation.

Several types of bias could influence methodological quality. Selection bias in non-randomized studies and omission of blinding participants or personnel could lead to increased motivation and more positive study results. Although it is almost impossible to perform blinding during physical interventions, one study was able to apply this on outcome assessors and patients (Granger et al., 2013). Attrition bias could have influenced results, although most patients indicated a reason for withdrawal. Nevertheless, some were unknown and could disguise potential negative treatment effects such as worse physical functioning or fatigue (Ni et al., 2016; Pouwels et al., 2015). Selective reporting is a concern as data regarding statistical significance were not always displayed for the home-based component specifically. Yet, evaluating whether there was a clinically relevant improvement in cardiorespiratory fitness after ( $p$ )rehabilitation was possible for all studies using the $6 \mathrm{MWT}$ distance. As only physical fitness was included in the search strategy, missing values for adherence treatment tolerance, and recovery were foreseen.

With regard to therapeutic validity, small sample sizes and patient cohort studies allow researchers to provide a flexible training scheme which can be easily monitored and adapted, thereby increasing patient and exercise adherence (Dalal et al., 2010; Pouwels et al., 2015). As a result, effect rates could be based on individually optimized, but slightly incomparable exercise programs (Hoogeboom et al., 2012; Herbert and Bø, 2005). Mainly larger studies disregard components like personalization of exercises and supervision for increasing quality and effectiveness of the intervention. Absolute training effects are more easy to compare in non-personalized fully structured training sessions (Thorsen et al., 2005), resulting in higher methodological quality. However, the main goal of home-based (p)rehabilitation should be to enhance and preserve wellbeing and everyday life in patients with NSCLC (Salander and Lilliehorn, 2016). Therefore, a patient tailored program with a necessity-based design should be at best interest, together with the highest therapeutical and methodological quality (Gridelli et al., 2007; Kilari et al., 2016). Furthermore, all patients with NSCLC can benefit from increasing endurance and muscle strength in order to optimize treatment outcomes by home-based (p)rehabilitation. However, the selection of patients should mainly focus on older and high-risk patients and how to increase accessibility to home-based, supervised, and personalized sessions in order to maximize training, physical fitness, adherence, treatment tolerance, and recovery (Kilari et al., 2016).

In conclusion, this systematic review showed positive and encouraging results of (home-based) (p)rehabilitation on physical fitness for patients with NSCLC. Although included studies varied in quality, and quantity, results of this review indicate that combining (home-based) resistance and endurance training, as well as supervision and personalization, seem necessary to optimize physical fitness, adherence, treatment tolerance, and recovery. Although different training contexts have been included in this review, home-based training alone has not been studied extensively and several studies were underpowerd. Therefore, additional randomized controlled trials are required. This sets priority for prospective trials including older and high-risk patients with NSCLC, in which supervision, personalization, and high methodological and therapeutic quality in a home-based context are investigated. Ultimately, more evidence for home-based (p)rehabilitation can be gathered leading to improved physical fitness, patient adherence, exercise adherence, and especially recovery and treatment tolerance in this predominantly high-risk patient group.

\section{Conflict of interest}

None.

\section{Acknowledgements}

The responsibility for the content of the manuscript is taken by ED. Both ED and MP have made substantial contributions to the acquisition of data, analysis and interpretation. All authors contributed to the interpretation and critical revision of the contents of the manuscript, provided approval for the current manuscript version, and agreed to be accountable for all aspects of the work and remaining questions. This research did not receive any specific grants from funding agencies in the public, commercial, or not-for-profit sectors.

\section{References}

Altenburg, W.A., Duiverman, M.L., ten Hacken, N.H.T., Kerstjens, H.A.M., de Greef, M.H.G., Wijkstra, P.J., et al., 2015. Changes in the endurance shuttle walk test in COPD patients with chronic respiratory failure after pulmonary rehabilitation: the minimal important difference obtained with anchor- and distribution-based method. Respir. Res. 16 (1), 27.

Andersen, A.H., Vinther, A., Poulsen, L.-L., Mellemgaard, A., 2011. Do patients with lung cancer benefit from physical exercise? Acta Oncol. 50 (2), 307-313.

Andersen, A.H. Vinther, A., Poulsen, L-L., Mellemgaard, A., 2013. A modified exercise protocol may promote continuance of exercise after the intervention in lung cancer patients-a pragmatic uncontrolled trial. Support. Care Cancer 21 (8), 2247-2253.

Arbane, G., Tropman, D., Jackson, D., Garrod, R., 2011. Evaluation of an early exercise intervention after thoracotomy for non-small cell lung cancer (NSCLC), effects on quality of life, muscle strength and exercise tolerance: randomised controlled trial. Lung Cancer 71 (2), 229-234.

Arbane, G., Douiri, A., Hart, N., Hopkinson, N.S., Singh, S., Speed, C., et al., 2014. Effect of postoperative physical training on activity after curative surgery for non-small cell lung cancer: a multicentre randomised controlled trial. Physiotherapy 100 (2), 100-107.

Bade, B.C., Thomas, D.D., Scott, J.B., Silvestri, G.A., 2015. Increasing physical activity and exercise in lung cancer: reviewing safety, benefits, and application. J. Thorac. Oncol. 10 (6), 861-871.

Brocki, B.C., Andreasen, J., Nielsen, L.R., Nekrasas, V., Gorst-Rasmussen, A. Westerdahl, E., 2014. Short and long-term effects of supervised versus unsupervised exercise training on health-related quality of life and functional outcomes following lung cancer surgery - a randomized controlled trial. Lung Cancer 83 (1), 102-108.

Cardenal, F., Nadal, E., Jové, M., Faivre-Finn, C., 2015. Concurrent systemic therapy with radiotherapy for the treatment of poor-risk patients with unresectable stage III non-small-cell lung cancer: a review of the literature. Ann. Oncol. 26 (2), 278-288.

Carli, F., Zavorsky, G.S., 2005. Optimizing functional exercise capacity in the elderly surgical population. Curr. Opin. Clin. Nutr. Metab. Care 8 (1).

Cavalheri, V., Tahirah, F., Nonoyama, M., Jenkins, S., Hill, K., 2014. Exercise training for people following lung resection for non-small cell lung cancer - a cochrane systematic review. Cancer Treat. Rev, 40 (4), 585-594.

Cheville, A.L., Kollasch, J., Vandenberg, J., Shen, T., Grothey, A., Gamble, G., et al., 2013. A home-based exercise program to improve function, fatigue, and sleep quality in patients with stage IV lung and colorectal cancer: a randomized controlled trial. J. Pain Symptom Manage. 45 (5), 811-821.

Chou, C.-H., Hwang, C.-L., Wu, Y.-T., 2012. Effect of exercise on physical function, daily living activities, and quality of life in the frail older adults: a meta-analysis. Arch. Phys. Med. Rehabil. 93 (2), 237-244.

Coats, V., Maltais, F., Simard, S., Fréchette, É., Tremblay, L., Ribeiro, F., et al., 2013. Feasibility and effectiveness of a home-based exercise training program before lung resection surgery. Can. Respir. J. 20 (2), e10-e16. 
Cochrane Statistical Methods Group and Cochrane Bias Methods Group, 2011a. Chapter 8: Assessing Risk of Bias in Included Studies [Nov 20th 2015] Available from: http://handbook.cochrane.org/.

Cochrane Statistical Methods Group and Cochrane Bias Methods Group, 2011b. Chapter 7: Selecting Studies and Collecting Data [Nov 20th 2015]; Available from: http://handbook.cochrane.org/.

Dalal, H.M., Zawada, A., Jolly, K., Moxham, T., Taylor, R.S., 2010. Home based versus centre based cardiac rehabilitation: cochrane systematic review and meta-analysis. BMJ 340.

Eberhardt, W.E.E., De Ruysscher, D., Weder, W., Le Péchoux, C., De Leyn, P., Hoffmann, H., et al., 2015. 2nd ESMO Consensus Conference in Lung Cancer: locally advanced stage III non-small-cell lung cancer. Ann. Oncol. 26 (8), 1573-1588.

Glotzer, O.S., Fabian, T., Chandra, A., Bakhos, C.T., 2013. Non-small cell lung cancer therapy: safety and efficacy in the elderly. Drug Healthcare Patient Saf. 5 113-121.

Granger, C.L., Chao, C., McDonald, C.F., Berney, S., Denehy, L., 2013. Safety and feasibility of an exercise intervention for patients following lung resection: a pilot randomized controlled trial. Integr. Cancer Ther. 12 (3), 213-224.

Granger, C.L., McDonald, C.F., Irving, L., Clark, R.A., Gough, K., Murnane, A., et al., 2014. Low physical activity levels and functional decline in individuals with lung cancer. Lung Cancer 83 (2), 292-299.

Granger, C.L., Holland, A.E., Gordon, I.R., Denehy, L., 2015. Minimal important difference of the 6-minute walk distance in lung cancer. Chronic Respir. Dis. 12 (2), 146-154.

Gridelli, C., Langer, C., Maione, P., Rossi, A., Schild, S.E., 2007. Lung cancer in the elderly. J. Clin. Oncol. 25 (14), 1898-1907.

Herbert, R.D., Bø, K., 2005. Analysis of quality of interventions in systematic reviews. Br. Med. J. 331 (7515), 507-509.

Hoffman, A.J., Brintnall, R.A., von Eye, A., Jones, L.W., Alderink, G., Patzelt, L.H., et al., 2014. A rehabilitation program for lung cancer patients during postthoracotomy chemotherapy. OncoTargets Ther. 7, 415-423.

Hoogeboom, T.J., Oosting, E., Vriezekolk, J.E., Veenhof, C., Siemonsma, P.C., de Bie, R.A., et al., 2012. Therapeutic validity and effectiveness of preoperative exercise on functional recovery after joint replacement: a systematic review and meta-analysis. PLoS ONE 7 (5), pe38031.

Hoogeboom, T.J., Dronkers, J.J., Hulzebos, E.H.J., van Meeteren, N.L.U., 2014. Merits of exercise therapy before and after major surgery. Curr. Opin. Anaesthesiol. 27 (2), 161-166.

Houchen-Wolloff, L., Boyce, S., Singh, S., 2015. The minimum clinically important improvement in the incremental shuttle walk test following cardiac rehabilitation. Eur. J. Prev. Cardiol. 22 (8), 972-978.

Hsu, C.-L., Chen, J.-H., Chen, K.-Y., Shih, J.-Y., Yang, J.C.-H., Yu, C.-J., et al., 2015. Advanced non-small cell lung cancer in the elderly: the impact of age and comorbidities on treatment modalities and patient prognosis. J. Geriatr. Oncol. 6 (1) $38-45$.

Jack, S., West, M., Grocott, M.P.W., 2011. Perioperative exercise training in elderly subjects. Best Pract. Res. Clin. Anaesthesiol. 25 (3), 461-472.

Janssen-Heijnen, M.L.G., Smulders, S., Lemmens, V.E.P.P., Smeenk, F.W.J.M., van Geffen, H.J.A.A., Coebergh, J.W.W., 2004. Effect of comorbidity on the treatment and prognosis of elderly patients with non-small cell lung cancer. Thorax 59 (7), 602-607.

Kale, M.S., Mhango, G., Gomez, J.E., Sigel, K., Smith, C.B., Bonomi, M., Wisnivesky, J.P., 2015. Treatment Toxicity in Elderly Patients with Advanced Non-Small Cell Lung Cancer. Am. J. Clin. Oncol., http://dx.doi.org/10.1097/COC. 0000000000000188 , Advance online publication.

Karvinen, K., Vallance, J., Walker, P., 2016. Newly diagnosed lung cancer patients' preferences for and beliefs about physical activity prior to chemotherapy. Psychol. Health Med. 21 (5), 593-600.

Kilari, D., Soto-Perez-de-Celis, E., Mohile, S.G., Alibhai, S.M.H., Presley, C.J., Wildes, T.M., et al., 2016. Designing exercise clinical trials for older adults with cancer: recommendations from 2015 Cancer and Aging Research Group NCI U13 meeting. J. Geriatr. Oncol. 7 (4), 293-304.

Kuehr, L.E.A., Wiskemann, J., Abel, U., Ulrich, C.M., Hummler, S., Thomas, M., 2014. Exercise in patients with non-small cell lung cancer. Med. Sci. Sports Exerc. 46 (4).

Laviolette, L., Bourbeau, J., Bernard, S., Lacasse, Y., Pepin, V., Breton, M.-J., et al., 2008. Assessing the impact of pulmonary rehabilitation on functional status in COPD. Thorax 63 (2), 115-121.

Moher, D., Liberati, A., Tetzlaff, J., Altman, D., The PRISMA Group, 2009. Preferred reporting items for systematic reviews and meta-analyses: the PRISMA statement. PLoS Med. 6 (7), e1000097.

Myers, J., Prakash, M., Froelicher, V., Do, D., Partington, S., Atwood, J.E., 2002. Exercise capacity and mortality among men referred for exercise testing. N. Engl. J. Med. 346 (11), 793-801.
Netherlands Cancer Registry, 2011. Richtlijn Oncologische Revalidatie (cited 2016); Available from: http://www.oncoline.nl/oncologische-revalidatie. Netherlands Cancer Registry, 2016a. Incidence of Lung Cancer in the Netherlands According to Type of Histology (1990-2015) (cited 2016); Available from: http://cijfersoverkanker.nl/selecties/Dataset_1/img576b9713d1fe6.

Netherlands Cancer Registry, 2016b. Survival of Non-small Cell Lung Cancer in the Netherlands According to Stage (TNM 6th edition 2003-2009)., Available from: http://cijfersoverkanker.nl/selecties/dataset_2/img5845740c6b9b7.

Netherlands Cancer Registry, 2016c. Incidence of Non-small Cell Lung Cancer in the Netherlands According To Five-year Age Groups (2005-2015), Available from: http://cijfersoverkanker.nl/selecties/dataset_3/img572754f344c02.

Ni, H.-J., Pudasaini, B., Yuan, X.-T., Li, H.-F., Shi, L., Yuan, P., 2016. Exercise training for patients pre- and postsurgically treated for non-small cell lung cancer: a systematic review and meta-analysis. Integr. Cancer Ther.

Oosting, E., Jans, M.P., Dronkers, J.J., Naber, R.H., Dronkers-Landman, C.M., Appelman-de Vries, S.M., et al., 2012. Preoperative home-based physical therapy versus usual care to improve functional health of frail older adults scheduled for elective total hip arthroplasty: a pilot randomized controlled trial. Arch. Phys. Med. Rehabil. 93 (4), 610-616.

Petrie, A., Sabin, C., 2009. Assessing agreement. In: Medical Statistics at a Glance. Blackwell Publishing, West Sussex, pp. 118-121.

Pouwels, S., Fiddelaers, J., Teijink, J.A.W., Woorst, JFt., Siebenga, J., Smeenk, F.W.J.M., 2015. Preoperative exercise therapy in lung surgery patients: a systematic review. Respir. Med. 109 (12), 1495-1504.

Quist, M., Rørth, M., Langer, S., Jones, L.W., Laursen, J.H., Pappot, H., et al., 2012. Safety and feasibility of a combined exercise intervention for inoperable lung cancer patients undergoing chemotherapy: a pilot study. Lung Cancer 75 (2), 203-208.

Redelmeier, D.A., Bayoumi, A.M., Goldstein, R.S., Guyatt, G.H., 1997. Interpreting small differences in functional status: the six minute walk test in chronic lung disease patients. Am. J. Respir. Crit. Care Med. 155 (4), 1278-1282.

Royal Dutch Physiotherapy Society, 2008. KNGF-richtlijn chronische obstructieve longziekten (cited 2016); Available from: https://www.fysionetevidencebased.nl/index.php/component/kngf/richtlijnen/copd/ praktijkrichtlijn-4/inleiding/inleiding?Itemid=.

Salander, P., Lilliehorn, S., 2016. To carry on as before: a meta-synthesis of qualitative studies in lung cancer. Lung Cancer 99, 88-93.

Santanello, N.C., Zhang, J., Seidenberg, B., Reiss, T.F., Barber, B.L., 1999. What are minimal important changes for asthma measures in a clinical trial? Eur. Respir. J. 14 (1), 23-27.

Schild, S.E., Stella, P.J., Geyer, S.M., Bonner, J.A., McGinnis, W.L., Mailliard, J.A., et al., 2003. The outcome of combined-modality therapy for stage iii non-small-cell lung cancer in the elderly. J. Clin. Oncol. 21 (17), 3201-3206.

Semrau, S., Zettl, H., Hildebrandt, G., Klautke, G., Fietkau, R., 2014. Older patients with inoperable non-small cell lung cancer. Strahlenther. Onkol. 190 (12), 1125-1132.

Singh, F., Newton, R.U., Galvão, D.A., Spry, N., Baker, M.K., 2013. A systematic review of pre-surgical exercise intervention studies with cancer patients. Surg. Oncol. 22 (2), 92-104.

Spruit, M.A., Singh, S.J., Garvey, C., ZuWallack, R., Nici, L., Rochester, C., et al., 2013. An Official American Thoracic Society/European Respiratory Society statement: key concepts and advances in pulmonary rehabilitation. Am. J. Respir. Crit. Care Med. 188 (8), e13-e64.

Temel, J.S., Greer, J.A., Goldberg, S., Vogel, P.D., Sullivan, M., Pirl, W.F., et al., 2009. A structured exercise program for patients with advanced non-small cell lung cancer. J. Thorac. Oncol. 4 (5), 595-601.

The Cochrane Collaboration, 2011. Cochrane Handbook for Systematic Revies of Interventions [Nov 15th 2015]; Available from: www.cochrane-handbook.org.

Thorsen, L., Skovlund, E., Strømme, S.B., Hornslien, K., Dahl, A.A., Fosså, S.D., 2005. Effectiveness of physical activity on cardiorespiratory fitness and health-related quality of life in young and middle-aged cancer patients shortly after chemotherapy. J. Clin. Oncol. 23 (10), 2378-2388.

Vansteenkiste, J., Crinò, L., Dooms, C., Douillard, J.Y., Faivre-Finn, C., Lim, E., et al., 2014. 2nd ESMO Consensus Conference on Lung Cancer: early-stage non-small-cell lung cancer consensus on diagnosis, treatment and follow-up. Ann. Oncol. 25 (8), 1462-1474.

Westmaas, J.L., Newton, C.C., Stevens, V.L., Flanders, W.D., Gapstur, S.M., Jacobs, E.J., 2015. Does a recent cancer diagnosis predict smoking cessation? an analysis from a large prospective US cohort. J. Clin. Oncol. 33 (15), 1647-1652.

Wright, A., Cook, C., Baxter, G., Dockerty, J., Abbott, J., 2011. A comparison of 3 methodological approaches to defining major clinically important improvement of 4 performance measures in patients with hip osteoarthritis. J. Orthop. Sports Phys. Ther. 41 (5), 319-327. 